\title{
Capsaicin Induces Theta-Band Synchronization between Gustatory and Autonomic Insular Cortices
}

\author{
Mitsuru Saito, ${ }^{1 \star}$ Hiroki Toyoda, ${ }^{1 \star}$ Shinpei Kawakami, ${ }^{1,2 \star}$ Hajime Sato, ${ }^{1 \star}$ Yong Chul Bae, ${ }^{3}$ and Youngnam Kang ${ }^{1}$ \\ ${ }^{1}$ Department of Neuroscience and Oral Physiology, Osaka University Graduate School of Dentistry, Suita, Osaka 565-0871, Japan, ${ }^{2}$ Nourishment Function \\ Laboratory, Health Care Division, Morinaga \& Company, Ltd., Yokohama, Kanagawa 230-8504, Japan, and ${ }^{3}$ Department of Oral Anatomy and \\ Neurobiology, BK21, School of Dentistry, Kyungpook National University, Daegu 700-412, South Korea
}

In the insular cortex, the primary gustatory area caudally adjoins the primary autonomic area that is involved in visceral sensory-motor integration. However, it has not been addressed whether neural activity in the gustatory insula (Gu-I) is coordinated with that in the autonomic insula (Au-I). We have demonstrated that TRPV1 activation in Gu-I induces theta-band synchronization between Gu-I and $\mathrm{Au}-\mathrm{I}$ in rat slice preparations. Electron-microscopic immunohistochemistry revealed that TRPV1 immunoreactivity was much higher in $\mathrm{Gu}-\mathrm{I}$ than in $\mathrm{Au}-\mathrm{I}$, and was mostly detected in dendritic spines receiving asymmetrical synapses. Whole-cell voltage-clamp recordings revealed that, in Gu-I, capsaicin-induced currents in layer 3 (L3) pyramidal cells (PCs) displayed no apparent desensitization, while those in layer 5 (L5) PCs displayed $\mathrm{Ca}^{2+}$-dependent desensitization, suggesting that L3 and L5 PCs respond differentially to TRPV1 activation. Voltage-sensitive dye imaging demonstrated that TRPV1 activation in Gu-I can alter an optical response with a monophasic and columnar temporospatial pattern evoked within Gu-I into an oscillatory one extending over Gu-I and Au-I. Power and cross-power spectral analyses of optical responses revealed theta-band synchronization between Gu-I and Au-I. Whole-cell current-clamp recordings demonstrated that such theta-band waves were mediated by sustained rhythmic firings at 4 and $8 \mathrm{~Hz}$ in L3 and L5 PCs, respectively. These results strongly suggested that theta-band oscillatory neural coordination between Gu-I and Au-I was induced by two distinct TRPV1mediated theta-rhythm firings in L3 and L5 PCs in Gu-I. This network coordination induced by TRPV1 activation could be responsible for autonomic responses to tasting and ingesting spicy foods.

\section{Introduction}

In the insular cortex, the rostral dysgranular region is involved in taste perception as the primary gustatory area (Yamamoto, 1987; Accolla et al., 2007), while its caudal granular region is potentially involved in visceral sensory-motor control as the primary autonomic area (Ruggiero et al., 1987; Cechetto and Saper, 1990; Yasui et al., 1991). Thus, the gustatory insula (Gu-I) and autonomic insula (Au-I) adjoin rostrocaudally. However, it has not been addressed whether neural activity in Gu-I is coordinated with that in Au-I.

When we taste and ingest hot and spicy foods containing capsaicin, we experience various autonomic responses such as perspiration from the face (Lee, 1954), salivation (Dunér-Engström et al., 1986), and facilitation of cardiovascular activity (Hachiya et

\footnotetext{
Received Dec. 31, 2011; revised July 31, 2012; accepted Aug. 7, 2012

Author contributions: Y.K. designed research; M.S., H.T., S.K., H.S., and Y.C.B. performed research; M.S., H.T., and H.S. analyzed data; Y.K. wrote the paper.

This work was supported by a donation from Morinaga \& Company, Ltd., and Grant-in-Aid for Scientific Research (B) 22300127 from the Japanese Ministry of Education, Culture, Sports, Science and Technology (Y.K.), and also supported by Grant for the Basic Science Research Program 2010-0029460 through the National Research Foundation from the Korean Ministry of Education, Science and Technology (Y.C.B.).

The authors declare no competing financial interests.

*M.S., H.T., S.K., and H.S. contributed equally to this work.

Correspondence should be addressed to Dr. Youngnam Kang, Department of Neuroscience and Oral Physiology, Osaka University Graduate School of Dentistry, 1-8, Yamadaoka, Suita, 0saka 565-0871, Japan. E-mail: kang@dent.osaka-u.ac.jp.

DOI:10.1523/JNEUROSCI.5906-11.2012

Copyright $\odot 2012$ the authors $\quad 0270-6474 / 12 / 3213470-18 \$ 15.00 / 0$
}

al., 2007). Although the exact mechanisms remain unknown, these responses are believed to be induced as a result of viscerovisceral autonomic reflexes (Ganong, 2003). Such reflexes might be caused by impulse activity in oral and gastrointestinal nociceptive afferents resulting from TRPV1 activation by capsaicin in a temperature/pH-dependent manner (Caterina et al., 1997; Tominaga et al., 1998). However, viscero-visceral reflexes might not preclude the possible involvement of higher-order sensorymotor integration between Au-I and Gu-I. Thus, the anatomical arrangement of $\mathrm{Gu}-\mathrm{I}$ and $\mathrm{Au}-\mathrm{I}$ together with such autonomic responses to spicy taste led us to investigate whether there exists coordination of neural activity between Gu-I and Au-I.

Although TRPV1 expression in rats was much lower in the cerebral cortex than in nociceptive DRG neurons (Sanchez et al., 2001), the highest TRPV1 expression in the mouse CNS was found in the agranular insular cortex (Roberts et al., 2004), which integrates pain information from peripheral nociceptive neurons (Treede et al., 1999; Jasmin et al., 2004). The mouse granular insular cortex displays similarly high TRPV1 expression (Roberts et al., 2004), but the detailed TRPV1 distribution in its subregions, dysgranular $\mathrm{Gu}-\mathrm{I}$ and granular $\mathrm{Au}-\mathrm{I}$, remains unknown.

In the rat $\mathrm{Gu}-\mathrm{I}$, there are a variety of neurons responding to gustatory, tactile, thermal, or nociceptive stimuli applied to the tongue and oral mucosa (Yamamoto et al., 1988; Hanamori et al., 1998) that express TRPV1 (Ishida et al., 2002; Kido et al., 2003). TRPV1 may also be expressed in thermal and/or nociceptive neurons in Gu-I. An fMRI study in human subjects has demonstrated 
that the tasting and swallowing of capsaicin cause excitation in Gu-I (Rudenga et al., 2010), suggesting that Gu-I processes the sensory information of hot and spicy taste of capsaicin. It can therefore be hypothesized that TRPV1 in Gu-I is involved in the perception of hot and spicy taste, similar to the putative important role of TRPV1 in the agranular insular cortex in central pain processing.

In the present study, we first investigated whether TRPV1 is differentially distributed in Gu-I and Au-I and subsequently examined whether the activation of TRPV1 in Gu-I induces neural coordination between $\mathrm{Gu}-\mathrm{I}$ and $\mathrm{Au}-\mathrm{I}$. Using voltage-sensitive dye imaging and whole-cell recording, we found that theta-band synchronization between $\mathrm{Gu}-\mathrm{I}$ and $\mathrm{Au}-\mathrm{I}$ was induced by two distinct TRPV1-mediated theta-rhythm firings in supragranular and infragranular pyramidal cells in Gu-I.

\section{Materials and Methods}

All experiments were performed in accordance with the guidelines of the animal ethics committees of our institutions for the care and use of laboratory animals.

Electron-microscopic immunohistochemistry. Three male Sprague Dawley rats weighing $300-320 \mathrm{~g}$ were used for this study. The rats were deeply anesthetized with sodium pentobarbital $(80 \mathrm{mg} / \mathrm{kg}$, i.p.) and perfused transcardially with $100 \mathrm{ml}$ of heparinized normal saline followed by 500 $\mathrm{ml}$ of freshly prepared mixture of $4 \%$ paraformaldehyde and $0.01 \%$ glutaraldehyde in $0.1 \mathrm{~m}$ phosphate buffer (PB), $\mathrm{pH}$ 7.4. Cerebral cortex including insula between 2.0 and $-0.8 \mathrm{~mm}$ from bregma was removed and postfixed in the same fixative for $2 \mathrm{~h}$ at $4^{\circ} \mathrm{C}$. Sections were cut transversely on a Vibratome at $60 \mu \mathrm{m}$ and cryoprotected in $30 \%$ sucrose in PB overnight at $4^{\circ} \mathrm{C}$.

Sections were frozen on dry ice for $20 \mathrm{~min}$ and thawed in $0.01 \mathrm{M} \mathrm{PBS}$, $\mathrm{pH} 7.2$, to enhance penetration, and pretreated with $1 \%$ sodium borohydride for $30 \mathrm{~min}$ to remove glutaraldehyde. Sections were then blocked with $3 \% \mathrm{H}_{2} \mathrm{O}_{2}$ for $10 \mathrm{~min}$ to suppress endogenous peroxidases, and with $10 \%$ normal donkey serum (Jackson ImmunoResearch) for $30 \mathrm{~min}$ to mask secondary antibody binding sites. For single immunostaining for TRPV1, sections were incubated overnight in goat anti-TRPV1 antibody (1:300; sc-12498; Santa Cruz Biotechnology) at $4^{\circ} \mathrm{C}$ and, the next day, rinsed in PBS for 15 min and incubated for $2 \mathrm{~h}$ in biotinylated donkey anti-goat antibody (1:200; Jackson ImmunoResearch). After washing, the sections were incubated with ExtrAvidin peroxidase (1:5000; SigmaAldrich) for $1 \mathrm{~h}$, and the immunoperoxidase was visualized by nickelintensified 3,3'-diaminobenzidine tetrahydrochloride (DAB). Sections were osmicated in osmium tetroxide (1\% in PB) for $1 \mathrm{~h}$, dehydrated in graded alcohols, flat embedded in Durcupan ACM Fluka (SigmaAldrich) between strips of ACLAR plastic film (Electron Microscopy Sciences), and cured for $48 \mathrm{~h}$ at $60^{\circ} \mathrm{C}$. Trapezoidal chips in superficial or deep layer of the insular cortex were cut out of the wafers and glued onto blank resin blocks with cyanoacrylate (see Fig. $2 A a, A b$ ). Thin sections were cut with a diamond knife, mounted on Formvar-coated single-slot nickel grids and stained with uranyl acetate and lead citrate. Grids were examined on an electron microscope (H-7500; Hitachi High Technologies). Images were captured by a cooled CCD camera (SC1000; Gatan). The specificity of primary antibody was confirmed by omitting the primary antibody in processing sections and by examining the consistency of immunostaining in adjacent serial thin sections of the same dendrites or terminals. Staining was eliminated by preincubation of the diluted antiserum (1:500) with blocking peptide (sc12498P; Santa Cruz Biotechnology) at a concentration of $46 \mu \mathrm{g} / \mathrm{ml}$. We also observed no staining with this antibody on material from TRPV1 ${ }^{-1-}$ mice (The Jackson Laboratory).

To assess the density of TRPV1-immunopositive neuropils, each of three randomly selected areas $\left(839.1 \mu \mathrm{m}^{2}\right)$ in respective layer $2 / 3$ and layer 5 of the insular cortex in a rat was subdivided into a matrix of $5 \times 6$ small square areas, from which 30 electron micrographs $\left(27.97 \mu \mathrm{m}^{2}\right.$ each; $30,000 \times)$ were captured. Numbers of TRPV1-immunopositive neuropils in electron micrograph were counted and summed over the 30 electron micrographs in each area, and the respective numbers of TRPV1-immunopositive neuropils obtained from the nine areas (three randomly selected areas in each of the three rats) were averaged. The density of TRPV1-immunopositive neuropils was defined as the number of TRPV1-immunopositive neuropils per $1000 \mu \mathrm{m}^{2}$.

Slice preparation. Wistar rats of both sexes at 14-22 d of age were used. Coronal sections of the Gu-I were cut at $300 \mu \mathrm{m}$ thick for whole-cell voltage-clamp recording. For voltage-sensitive dye (VSD) imaging and whole-cell current-clamp recording, slice preparations, namely tiltedhorizontal slices, were cut along the rhinal fissure (RF) in a plane tilted up at $15^{\circ}$ to the horizontal plane at $350 \mu \mathrm{m}$ thick, to include the agranular insula (AI), the dysgranular insula (DI), and the granular insula (GI) by referring to the middle cerebral artery (MCA) (Fig. 1) (Accolla et al., 2007). GI has recently been reclassified as GI/DI (Paxinos and Watson, 1998). DI represents the gustatory area (Gu-I), and GI/DI represents the autonomic area $(\mathrm{Au}-\mathrm{I})$. Caudal end of $\mathrm{Au}-\mathrm{I}$ is located at $2.0 \mathrm{~mm}$ caudal to MCA (Cechetto and Saper, 1987). In the experiments using VSD imaging or whole-cell current-clamp recording, all the data were obtained from the tilted-horizontal slices, the bottom surface of which was located 350 $\mu \mathrm{m}$ dorsal to RF. When three serial sections of $100 \mu \mathrm{m}$ thickness cut at the levels between 350 and $650 \mu \mathrm{m}$ dorsal to RF were examined using Nissl staining in the 10 rats examined, the boundary between DI and GI/DI that was determined based on the thickness of the granular layer was consistently found to be between 500 and $750 \mu \mathrm{m}$ caudal to MCA (Fig. $1 \mathrm{Ca}$ ). Similarly, in the living slice preparations of $350 \mu \mathrm{m}$ thickness, the boundary between Gu-I and Au-I could also be determined based on the thickness of layer 4 that is discernible as a differentially shaded region between layers $2 / 3$ and 5 in either bright-field or resting-light-intensity images. Such a boundary in a living slice was confirmed to be almost the same as that determined in the Nissl-stained image (Fig. $1 D$ ), which was obtained from the same slice processed for Nissl staining after the slice was fixed overnight in $4 \%$ paraformaldehyde and then sectioned on the frozen microtome at $50 \mu \mathrm{m}$.

The slices were placed in the recording chamber (volume, $1.0 \mathrm{ml}$ ), which was perfused with artificial CSF (ACSF) (in mM: $126 \mathrm{NaCl}, 3 \mathrm{KCl}$, $1 \mathrm{MgCl}_{2}, 1.25 \mathrm{NaH}_{2} \mathrm{PO}_{4}, 26 \mathrm{NaHCO}_{3}, 2 \mathrm{CaCl}_{2}$, and 10 D-glucose; $\mathrm{pH} 7.3$ ) at a flow rate of $1.5 \mathrm{ml} / \mathrm{min}(=25 \mu \mathrm{l} / \mathrm{s})$ at room temperature $\left(20-24^{\circ} \mathrm{C}\right)$. ACSF was continuously gassed with $95 \% \mathrm{O}_{2}-5 \% \mathrm{CO}_{2}$.

Whole-cell recordings. The procedures for the whole-cell recordings were previously described (Kang et al., 2007). Whole-cell recordings were obtained from layer 3 (L3) and layer 5 (L5) neurons whose pyramidal shape and proximal apical dendrites were clearly seen at $60 \times$ objective under infrared-differential interference contrast microscopy. When recording the current response to capsaicin puff application under voltage-clamp condition, $1 \mu \mathrm{M}$ tetrodotoxin, $100 \mu \mathrm{M} \mathrm{Ni}{ }^{2+}$, and $500 \mu \mathrm{M}$ $\mathrm{Cd}^{2+}$ were added in the extracellular solution, in which concentrations of divalent cations were modified in two ways: one containing $0.5 \mathrm{~mm}$ $\mathrm{Ca}^{2+}$ and $2.5 \mathrm{mM} \mathrm{Mg}^{2+}$, and the other containing $2 \mathrm{mM} \mathrm{Ca}^{2+}$ and $1 \mathrm{~mm}$ $\mathrm{Mg}^{2+}$. The internal solution had the following composition (in mM): 140 CsCl, 10 HEPES-Na, 2 ATP-Mg, 0.3 GTP-Na 3 , 10 EGTA; pH 7.3, adjusted with $\mathrm{CsOH}$. The patch pipettes had a DC resistance of 4-5 M $\Omega$ when filled with the internal solution. The sealing resistance was usually $>10 \mathrm{G} \Omega$. Under the voltage-clamp condition at the holding potential of $-60 \mathrm{mV}$, a depolarizing ramp pulse from -100 to $+50 \mathrm{mV}$ for $1 \mathrm{~s}$ was applied during $5 \mathrm{~s}$ puff application of $20 \mu \mathrm{M}$ capsaicin that was repeated every $25 \mathrm{~s}$. Respective amplitudes of inward currents $\left(I_{\text {cap }}\right)$, evoked by repetitive puff applications of capsaicin, were measured from the baseline current obtained before puff applications. The conductance was measured using linear regression across the linear part of the currentvoltage plot $(-90$ to $-20 \mathrm{mV}$ ) in response to the ramp pulse. For pooled analysis, respective amplitudes of $I_{\text {cap }}$ were normalized to that of the first one, and the conductances $\left(G_{\text {cap }}\right)$ obtained during capsaicin puff application were normalized to the control conductance. For the currentclamp recordings, the internal solution had the following composition (in mM): $123 \mathrm{~K}$-gluconate, $18 \mathrm{KCl}, 14 \mathrm{NaCl}, 2 \mathrm{ATP}-\mathrm{Mg}, 0.3 \mathrm{GTP}-\mathrm{Na}_{3}, 10$ HEPES, 0.1 EGTA; pH 7.3, adjusted with KOH. Microstimulation (100 $\mu$ s duration) was delivered via a sharp monopolar tungsten electrode (DC resistance, $0.8-1.8 \mathrm{M} \Omega$ ) placed at a site $150-450 \mu \mathrm{m}$ rostral to $\mathrm{MCA}$ in layer 4 of Gu-I. Whole-cell currents or voltages were recorded 

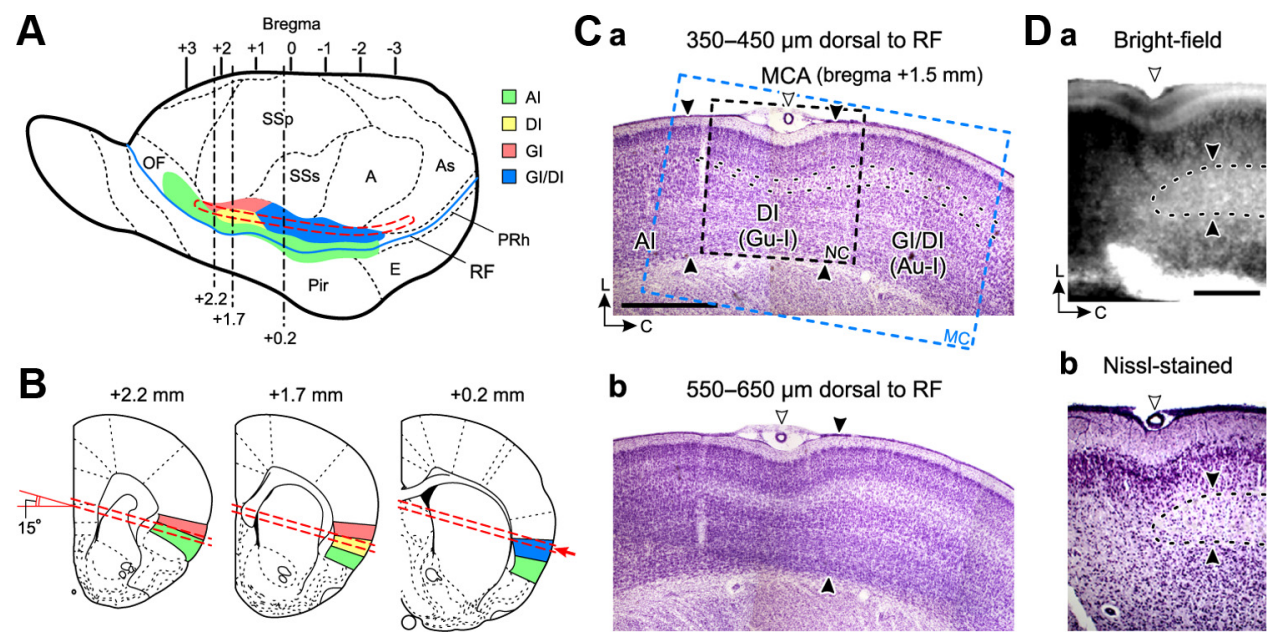

Figure 1. Locations of gustatory and autonomic insular cortices in coronal and horizontal sections. A, A lateral view of rat whole brain modified from a report (Cechetto and Saper, 1987), in which respective regions of the insular cortex are colored differentially. Agranular insula (Al), green; dysgranular insula (DI), yellow; granular insula (GI), orange; hybrid region of Gl and DI (GI/DI), blue. Slice preparations were obtained from the region demarcated with a red interrupted line. The blue continuous curve indicates the rhinal fissure (RF). A, Auditory cortex; As, association cortex; E, entorhinal cortex; OF, orbitofrontal cortex; Pir, piriform cortex; PRh, perirhinal cortex; SSp, primary somatosensory cortex; SSs, secondary somatosensory cortex. B, Cross sections of a horizontal slice (red interrupted lines) of the insular cortex at respective levels of coronal brain sections from bregma (Paxinos and Watson, 1998) (see Materials and Methods). C, Microphotographs of Nissl-stained sections of the tilted-horizontal slice (350 $\mu \mathrm{m}$ thick) of the rat insular cortex, at the levels of 350-450 $\mu \mathrm{m}(\boldsymbol{a})$ and $550-650 \mu \mathrm{m}(\boldsymbol{b})$ dorsal to RF. An open arrowhead indicates MCA. Three distinct regions of the insular cortex were identified based on the thickness of the granular layer, which is demarcated with black interrupted curves. Scale bar, $1 \mathrm{~mm}$. The two pairs of filled facing arrowheads indicate the borders between Al and DI (left pair) and between DI and GI/DI (right pair). The rectangular areas enclosed with black (NC) and blue (MC) interrupted lines indicate the captured areas of voltage-sensitive dye imaging using NeuroCCD-sm (Figs. 5, 6) and MiCAM02-HR (Fig. 7), respectively. L, Lateral; $C$, caudal. $D$, The boundary (filled arrowheads) determined in the living slices using bright-field ( $\boldsymbol{a}$ ) was confirmed to be almost the same as that determined in the Nissl-stained image $(\boldsymbol{b})$. The bottom and top parts of the bright-field image were dissimilar from the corresponding parts of the Nissl image, due to an enhancement of contrast of the bright-field image.

with Axopatch 200B (Molecular Devices). Signals were low-pass filtered at $2 \mathrm{kHz}$ (four-pole Bessel filter) and digitized at a sampling rate of 2-10 $\mathrm{kHz}$ (Digidata 1322A or 1440A; Molecular Devices). The membrane potential values given in the text were corrected for the liquid junction potential $(10 \mathrm{mV})$ between the K-gluconate-based internal solution (negative) and the standard extracellular solution.

Optical recording using a VSD. The procedures for the optical recording and its data analysis were previously described (Sato et al., 2008). Briefly, the slices were stained with $200 \mu \mathrm{M}$ RH414 (Invitrogen). The slices were illuminated with light of $535 \pm 15 \mathrm{~nm}$ wavelength using a stabilized $150 \mathrm{~W}$ xenon lamp. The fluorescence emitted from VSD was long-pass filtered above $580 \mathrm{~nm}$ and measured with either of the two CCD cameras (NeuroCCD-sm; RedShirtImaging; or MiCAM02-HR; BrainVision), which was attached to a microscope (BX-51WI; Olympus) equipped with a water-immersion objective $(10 \times, 0.3 \mathrm{NA})$ or an air objective (XLFluor $4 \times / 340,0.28 \mathrm{NA}$; Olympus). The imaged area was $1.6 \times 1.6 \mathrm{~mm}^{2}$ (NeuroCCD) or $4.6 \times 3.1 \mathrm{~mm}^{2}$ (MiCAM), and each pixel (element) of the $80 \times 80$ array (NeuroCCD) or $184 \times 124$ array (MiCAM) detected the optical signals. The rectangular areas enclosed with black (NC) and blue (MC) interrupted lines indicate the captured areas of voltage-sensitive dye imaging using NeuroCCD (see Figs. 5, 6, 9) and MiCAM02-HR (see Figs. 7, 8), respectively (Fig. 1Ca). The fluorescence images were captured at a sampling rate of $125-1000 \mathrm{~Hz}$. Optical responses were analyzed by using NeuroPlex (RedShirtImaging) or BV_Ana (BrainVision), and were displayed as pseudocolor images. Eight series of 512 optical images obtained every $15 \mathrm{~s}$ were averaged to improve the signal-to-noise ratio when necessary. No change in fluorescence intensity was observed in the absence of RH414. The threshold current intensity for evoking a columnar spatial pattern of the optical response ranged between 3.2 and $3.7 \mu \mathrm{A}$ with a mean value of $3.4 \mu \mathrm{A}$ in coronal slices $(n=12)$ (Sato et al., 2008). This mean value was used as a presumed mean threshold $\left(\mathrm{Th}^{*}\right)$ in the horizontal slices, because the threshold would not be changed as long as the local circuit is preserved regardless of coronal or horizontal slice preparations. Effects of capsaicin were examined on the spatiotemporal pattern of excitation spread that was induced by stimulation with the current intensity of either 1.2 -fold $(4 \mu \mathrm{A})$ or 1.8 -fold $(6 \mu \mathrm{A})$ presumed mean threshold $\left(1.2 \times \mathrm{Th}^{*}\right.$ and $1.8 \times \mathrm{Th}^{*}$, respectively), to be consistent with our previous study (Sato et al., 2008).
Power and cross-power spectral analyses (Sklar et al., 1972) of temporal profiles of optical responses were performed using STATISTICA 10J (StatSoft).

Drug application. Capsaicin (Wako Pure Chemical) and 5'iodoresiniferatoxin (I-RTX) (Sigma-Aldrich) as an antagonist of TRPV1 were bath-applied at 5-10 and $0.5 \mu \mathrm{M}$, respectively. By using a pressurepulsed microinjector (6-8 psi; PV830; World Precision Instruments), 20 $\mu \mathrm{M}$ capsaicin was puff-applied for $5 \mathrm{~s}$ at a flow rate of $<0.15 \mu \mathrm{l} / \mathrm{s}$ through a glass pipette. The tip of the glass pipette was placed $50-100 \mu \mathrm{m}$ above the slice surface and was directed radially to the cortical surface and at an angle of $15-25^{\circ}$ to the slice surface to puff capsaicin over the somadendritic domain of the recorded cell. Considering the chamber volume $(1.0 \mathrm{ml})$ and the large difference in flow rate between the extracellular solution and the puff-applied capsaicin $(25 \mathrm{vs}<0.15 \mu \mathrm{l} / \mathrm{s})$, the concentration of puff-applied capsaicin at a given site on the soma-dendritic domain of the recorded cell would promptly reach a steady level at least several times smaller than $20 \mu \mathrm{M}$ after the onset of the $5 \mathrm{~s}$ puff. The concentrations of bath-applied capsaicin in this study were similar to those used in previous studies on TRPV1 in CNS neurons using slice preparations [ $\leq 10 \mu \mathrm{M}$ (Marinelli et al., 2003); $3 \mu \mathrm{M}$ (Grueter et al., 2010); $\leq 10 \mu \mathrm{M}$ (Cavanaugh et al., 2011)]. Such concentrations are reasonable in view of the dose-response relationship in cultured DRG neurons $\left(\mathrm{EC}_{50}=\right.$ $1.1 \mu \mathrm{M}$ ) (Kim et al., 2006). Phorbol 12-myristate 13-acetate (PMA) (SigmaAldrich) was bath-applied at $10 \mu \mathrm{M}$, as protein kinase C (PKC) activation by PMA either enhances the capsaicin-induced membrane currents (Vellani et al., 2001; Numazaki et al., 2002) or reverses the desensitization of TRPV1 responses (Mandadi et al., 2006).

Statistical analysis. Numerical data were expressed as the mean \pm $\mathrm{SD}$ or the geometric mean $\times[\text { geometric SD }]^{ \pm 1}$. The statistical significances in nonpairwise and pairwise experiments were assessed using unpaired $\left({ }^{\dagger}\right)$ and paired $\left({ }^{\ddagger}\right)$ Student's $t$ test, respectively, or factorial $\left({ }^{\$}\right)$ and repeated-measure $\left({ }^{\$}\right)$ ANOVA, respectively, followed by Fisher's protected least significant difference post hoc test $\left({ }^{\Pi}\right.$ ) (STATISTICA 10J; StatSoft; Mathematica 8; Wolfram Research). The two-sample Kolmogorov-Smirnov (K-S) test was used to compare two cumulative probability distributions. A value of $p<0.05$ was considered statistically significant. 


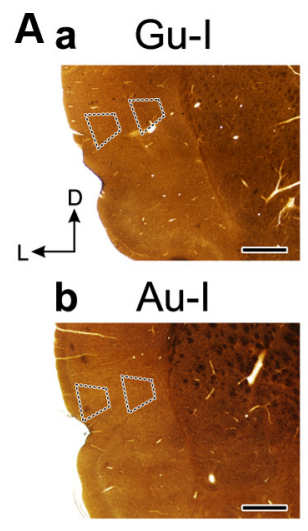

B $\quad$ Gu-I

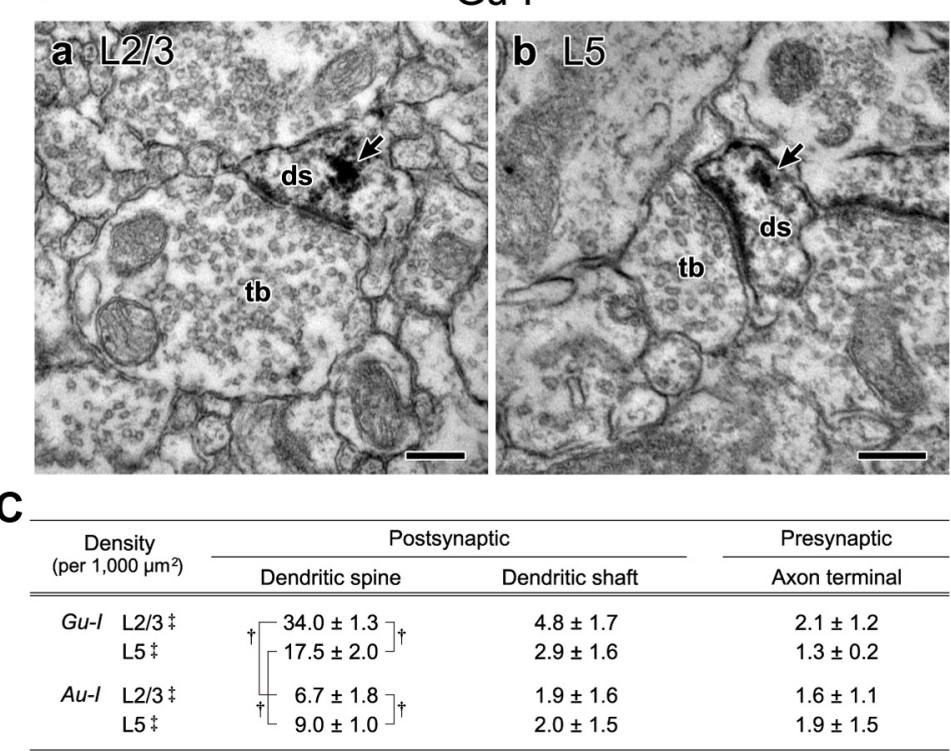

Figure 2. Expression of TRPV1 in the rat insular cortex. $\boldsymbol{A}$, Bright-field images of coronal sections of Gu-I (a) and Au-I $(\boldsymbol{b})$ at +1.5 and $-0.3 \mathrm{~mm}$ from bregma, respectively, in which immunoperoxidase-DAB immunostaining for TRPV1 was performed. The respective trapezoidal areas (dotted lines) localized in the superficial (L2/3) and deep (L5) layers of Gu-I and Au-I show the regions in which the density of TRPV1-immunopositive neuropils was analyzed using electron microscopy. Scale bar, $0.5 \mathrm{~mm}$. L, Lateral; D, dorsal. B, Electron micrographs showing TRPV1-positive postsynaptic dendritic spines (ds) in L2/3 (a) and L5 (b) of the rat Gu-I. Terminal boutons (tb) making asymmetrical synapses on TRPV1-positive dendritic spines (ds) in L2/3 and L5 of Gu-I. The arrows indicate electron-dense immunoreaction products. Scale bars, $200 \mathrm{~nm}$. C, Density (mean \pm SD, per $1000 \mu \mathrm{m}^{2}$ ) of TRPV1-immunopositive postsynaptic dendrites (spine and shaft) and presynaptic axon terminals in $L 2 / 3$ and $L 5$ of the rat insular cortex $(n=9) .{ }^{\dagger} p<0.02 ;{ }^{\ddagger} p<0.001$ (postsynaptic vs presynaptic).

\section{Results}

\section{Subcellular distribution of TRPV1 in Gu-I and Au-I}

Using an electron-microscopic immunohistochemical method, we first examined whether and how TRPV1 is distributed in Gu-I and $\mathrm{Au}-\mathrm{I}$ ( +1.5 and $-0.3 \mathrm{~mm}$ from bregma, respectively). In the respective trapezoidal areas in the supragranular and infragranular layers of Gu-I and Au-I (Fig. 2Aa,Ab), the distribution of TRPV1 was examined. In layers $2 / 3$ (L2/3) and 5 (L5) of Gu-I, TRPV1 immunoreactivity was mostly detected in postsynaptic dendritic spines receiving asymmetrical synapses (Fig. $2 \mathrm{Ba}, \mathrm{Bb}$ ). As summarized in a table (Fig. 2C), the density of TRPV1immunopositive dendritic spines (per $1000 \mu \mathrm{m}^{2}$; see Materials and Methods) was two times higher in L2/3 $\left(34.0 \pm 1.3 ;{ }^{\dagger} p<0.001\right)$ than in $\mathrm{L} 5(17.5 \pm 2.0)$ in Gu-I. Compared with Gu-I, Au-I expressed much lower TRPV1. The density of TRPV1-immunopositive dendritic spines in L2/3 was five times lower in Au-I $\left(6.7 \pm 1.8 ;{ }^{\dagger} p<\right.$ $0.001)$ than in Gu-I, while that in L5 was two times lower in Au-I $\left(9.0 \pm 1.0 ;{ }^{\dagger} p<0.001\right)$ than in Gu-I (Fig. $\left.2 C\right)$. Thus, TRPV1 was more densely expressed in the dendritic spines of pyramidal cells in $\mathrm{Gu}-\mathrm{I}$ than in those of pyramidal cells in Au-I.

\section{Capsaicin-induced currents in L3 and L5 PCs in Gu-I}

Next, we examined how L3 and L5 pyramidal cells (PCs) in Gu-I respond to puff application of capsaicin under the condition of either 0.5 or $2 \mathrm{mM}\left[\mathrm{Ca}^{2+}\right]_{\mathrm{o}}$. When $20 \mu \mathrm{M}$ capsaicin was puffapplied repeatedly to an L3 PC (Fig. $3 A a$ ), both capsaicininduced inward currents $\left(I_{\text {cap }}\right)$ and current responses to the ramp pulses from -100 to $+50 \mathrm{mV}$ for $1 \mathrm{~s}$ obtained at $2 \mathrm{mM}\left[\mathrm{Ca}^{2+}\right]_{\mathrm{o}}$ displayed no apparent desensitization (Fig. $3 A a, A b,{ }^{\star} 1$ and ${ }^{\star} 4$ ). The current responses to the ramp pulses obtained before and during puff applications of capsaicin crossed each other at $\sim 0$ $\mathrm{mV}$ (Fig. 3Ab, arrowhead), consistent with the nature of TRPV1 as a nonselective cation channel observed in HEK293 cells (Caterina et al., 1997). With repeated capsaicin puff applications, both the mean values of the peak $I_{\text {cap }}$ and of the conductance $\left(G_{\text {cap }}\right)$ increased in L3 PCs examined at $0.5 \mathrm{~mm}\left[\mathrm{Ca}^{2+}\right]_{\mathrm{o}}(n=5)$ as well as in those examined at $2 \mathrm{~mm}\left[\mathrm{Ca}^{2+}\right]_{\mathrm{o}}(n=5)$ (hollow and solid columns, respectively), while the facilitatory summations of $I_{\text {cap }}$ and subsequent increases in $G_{\text {cap }}$ were significantly larger $\left({ }^{\$} p<0.05\right.$ and ${ }^{\$} p<0.01$, respectively) at $2 \mathrm{~mm}\left[\mathrm{Ca}^{2+}\right]_{\mathrm{o}}$ than at $0.5 \mathrm{~mm}\left[\mathrm{Ca}^{2+}\right]_{\mathrm{o}}$ (Fig. 3Ac,Ad). This result was contrary to the $\mathrm{Ca}^{2+}$-dependent tachyphylaxis or desensitization of TRPV1 in rat DRG neurons (Koplas et al., 1997).

In contrast, in L5 PCs, the first puff application of capsaicin induced a large $I_{\text {cap }}$ that desensitized rapidly at $0.5 \mathrm{~mm}\left[\mathrm{Ca}^{2+}\right]_{\mathrm{o}}$ (Fig. $\left.3 B a, B b,{ }^{\star} 1\right)$. Subsequent capsaicin puff applications induced no prominent current responses, revealing tachyphylaxis in $I_{\text {cap }}$ and in $G_{\text {cap }}$ (Fig. $3 B a, B b,{ }^{\star} 3$ and ${ }^{\star} 5$ ). Although the difference in the tachyphylaxis of the normalized $I_{\text {cap }}$ between L5 PCs examined at $0.5 \mathrm{~mm}$ $\left[\mathrm{Ca}^{2+}\right]_{\mathrm{o}}(n=5)$ and those examined at $2 \mathrm{mM}\left[\mathrm{Ca}^{2+}\right]_{\mathrm{o}}(n=5)$ was not significant $\left({ }^{\$} p>0.1\right)$, the normalized second $I_{\text {cap }}$ was significantly smaller $\left({ }^{\dagger} p<0.05\right)$ at $2 \mathrm{mM}\left[\mathrm{Ca}^{2+}\right]_{\mathrm{o}}$ (solid column) than at $0.5 \mathrm{~mm}\left[\mathrm{Ca}^{2+}\right]_{\mathrm{o}}$ (hollow column) (Fig. $3 \mathrm{Bc}$ ). The difference in the tachyphylaxis of the normalized $G_{\text {cap }}$ was significantly larger $\left({ }^{\$} p<\right.$ 0.02 ) at $2 \mathrm{~mm}\left[\mathrm{Ca}^{2+}\right]_{\mathrm{o}}$ than at $0.5 \mathrm{~mm}\left[\mathrm{Ca}^{2+}\right]_{\mathrm{o}}$ (Fig. $3 B d$ ). These results suggest an enhancement of tachyphylaxis in $I_{\text {cap }}$ and in $G_{\text {cap }}$ by a $\left[\mathrm{Ca}^{2+}\right]_{0}$ increase. Furthermore, the mean half-duration $(0.6 \pm$ $0.6 \mathrm{~s} ; n=5)$ of the first $I_{\text {cap }}$ obtained at $2 \mathrm{mM}\left[\mathrm{Ca}^{2+}\right]_{\mathrm{o}}$ was significantly shorter $\left({ }^{\dagger} p<0.02\right)$ than that $(2.3 \pm 1.1 \mathrm{~s} ; n=5)$ at $0.5 \mathrm{~mm}$ $\left[\mathrm{Ca}^{2+}\right]_{0}$. These observations are consistent with the $\mathrm{Ca}^{2+}$. dependent desensitization of TRPV1 in rat DRG neurons (Koplas et al., 1997). Thus, the kinetic properties of $I_{\text {cap }}$ differ considerably between L3 and L5 PCs.

Effects of PKC activation on $I_{\text {cap }}$ in L3 and L5 PCs in Gu-I PKC activation either enhances the capsaicin-induced membrane currents in rat DRG neurons and heterologous expression systems (Vellani et al., 2001; Numazaki et al., 2002) or reverses 
A

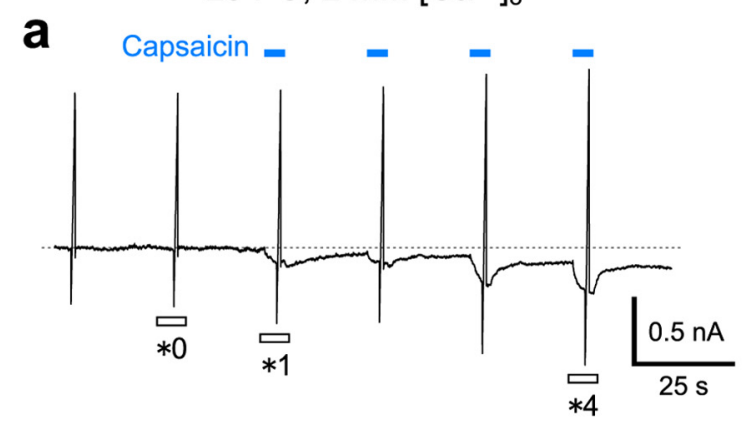

b

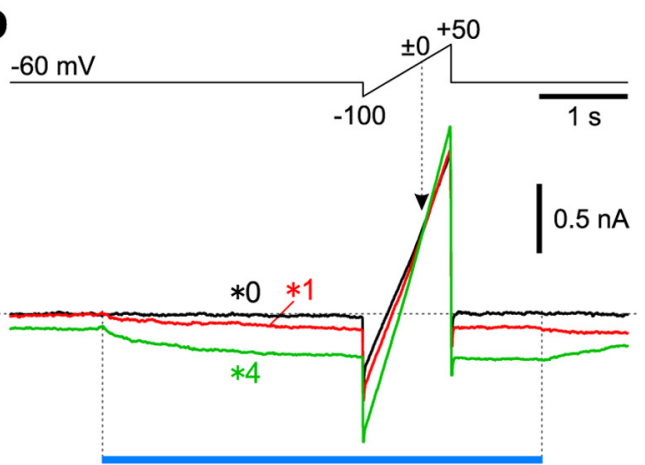

$20 \mu \mathrm{M}$ Capsaicin

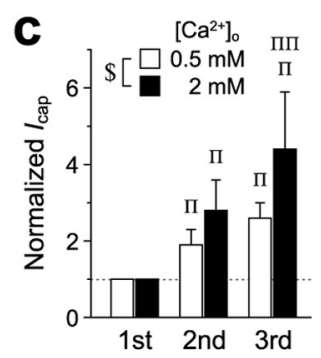

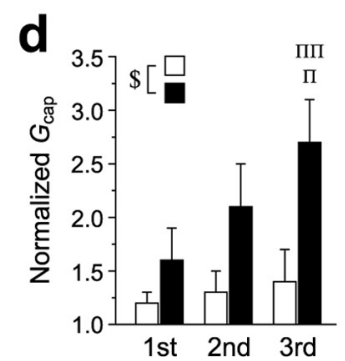

B
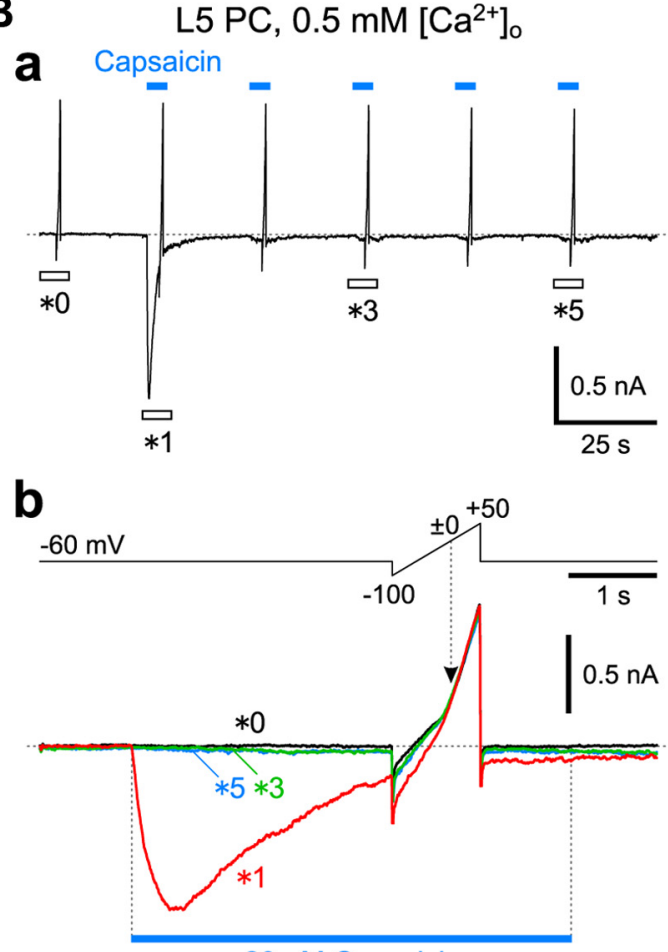

$20 \mu \mathrm{M}$ Capsaicin
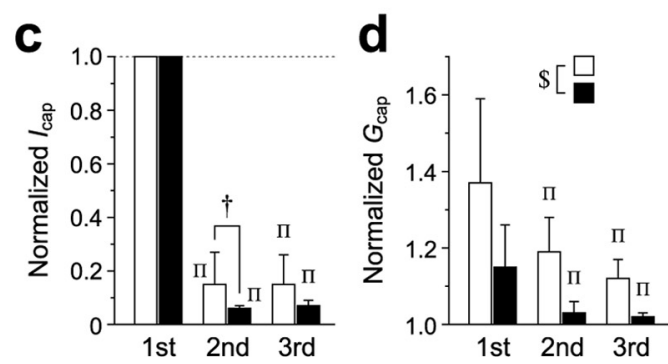

Figure 3. Capsaicin-induced currents in $\mathrm{L} 3$ and $L 5 \mathrm{PC}$ in Gu-I. Aa, Ba, Sample traces of continuous recordings of inward currents $\left(I_{\text {cap }}\right)$ induced by $5 \mathrm{~s}$ puff application of $20 \mu \mathrm{M}$ capsaicin and the current responses to the ramp pulses ( $\boldsymbol{A} \boldsymbol{b}, \boldsymbol{B} \boldsymbol{b}$, top) applied before and during respective puff applications of capsaicin (blue bars), obtained in an $\mathrm{L} 3 \mathrm{PC}$ at $2 \mathrm{~mm}\left[\mathrm{Ca}{ }^{2+}\right]_{0}(\boldsymbol{A})$ and an $\mathrm{L} 5 \mathrm{PC}$ at $0.5 \mathrm{~mm}$ $\left[\mathrm{Ca}^{2+}\right]_{0}(\boldsymbol{B})$. Holding potential, $-60 \mathrm{mV}$. $\boldsymbol{A} \boldsymbol{b}, \boldsymbol{B} \boldsymbol{b}$, Top, Positive ramp pulse from -100 to $+50 \mathrm{mV}$ for $1 \mathrm{~s}$ applied at the holding potential of $-60 \mathrm{mV}$. Bottom, Superimposed enlarged traces of current responses seen during the respective time periods indicated with open horizontal bars in $\boldsymbol{A a}\left({ }^{*} 0,{ }^{*} 1\right.$ and $\left.{ }^{*} 4\right)$ and $\boldsymbol{B a}\left({ }^{*} 0,{ }^{*} 1,{ }^{*} 3\right.$ and $\left.{ }^{*} 5\right)$. The blue bars indicate the duration and timing of puff applications of capsaicin. The current responses to the ramp pulse crossed each other at $\sim 0 \mathrm{mV}$ (arrowheads). $A c, B c$, Mean $(+S D)$ amplitudes of $I_{\text {cap }}$ obtained in response to the first through the third puff application of capsaicin, normalized to that obtained during the first puff application in $\mathrm{L} 3 \mathrm{PCs}$ at $0.5 \mathrm{~mm}\left[\mathrm{Ca}^{2+}\right]_{0}$ (hollow columns: second, $1.9 \pm 0.4 ;$ third, $2.6 \pm 0.4 ; n=5$ ) and in a different set of $\mathrm{L} 3 \mathrm{PCs}$ at $2 \mathrm{~mm}\left[\mathrm{Ca}^{2+}\right]_{0}$ (solid columns: second, $2.8 \pm 0.8 ;$ third, $\left.4.4 \pm 1.5 ; n=5\right)\left(\boldsymbol{A c}\right.$ ) and those in $\mathrm{L} 5 \mathrm{PCs}$ at $0.5 \mathrm{~mm}\left[\mathrm{Ca}^{2+}\right]_{0}$ (hollow columns: $0.15 \pm 0.12 ; 0.15 \pm 0.11 ; n=5$ ) and in a different set of $\mathrm{L} 5 \mathrm{PCs}$ at $2 \mathrm{~mm}\left[\mathrm{Ca}^{2+}\right]_{0}$ (solid columns: $\left.0.06 \pm 0.01 ; 0.07 \pm 0.02 ; n=5\right)(\boldsymbol{B C}) .{ }^{\dagger} p<0.05 ;{ }^{5} p<0.03 ;{ }^{\Pi} p<0.05$ (vs First); ${ }^{\Pi \Pi} p<0.05$ (vs Second). These symbols are the same as those applied in $\boldsymbol{A d}$ and $\boldsymbol{B d}$. $\boldsymbol{A d}, \boldsymbol{B d}$, Mean $(+S D)$ values of conductances $\left(G_{\text {cap }}\right)$ obtained in response to the first through the third puff application of capsaicin, normalized to that obtained in the control conditions in $\mathrm{L} 3 \mathrm{PCs}$ at $0.5 \mathrm{~mm}\left[\mathrm{Ca}^{2+}\right]_{0}$ (hollow columns: first, $1.2 \pm 0.1$; second, $1.3 \pm 0.2 ;$ third, $1.4 \pm 0.3 ; n=5$ ) and in the different set of $\mathrm{L} 3 \mathrm{PCs}$ at $2 \mathrm{mM}\left[\mathrm{Ca}^{2+}\right]_{0}$ ( solid columns: first, $1.6 \pm 0.3$; second, $2.1 \pm 0.3$; third, $2.7 \pm 0.3 ; n=5)(\boldsymbol{A d})$ and those in $\mathrm{L} 5 \mathrm{PC}$ at $0.5 \mathrm{~mm}\left[\mathrm{Ca}^{2+}\right]_{0}$ (hollow columns: $\left.1.37 \pm 0.22 ; 1.19 \pm 0.09 ; 1.12 \pm 0.05 ; n=5\right)$ and in the different set of $L 5 P C$ at $2 \mathrm{~mm}\left[\mathrm{Ca}^{2+}\right]_{0}$ (solid columns: $\left.1.15 \pm 0.11 ; 1.03 \pm 0.03 ; 1.01 \pm 0.01 ; n=5\right)(\boldsymbol{B d})$.

the desensitization of TRPV1 responses in rat DRG neurons and heterologous expression systems (Mandadi et al., 2004, 2006). Accordingly, we next investigated whether PKC activation is differentially involved in the modulation of the $I_{\text {cap }}$ between L3 and L5 PCs. As shown in Figure 3, $A c, A d$, and $B c, B d$, both the facilitatory summation of capsaicin-induced currents in L3 PCs and the tachyphylaxis or desensitization of those in L5 PCs were larger at $2 \mathrm{mM}\left[\mathrm{Ca}^{2+}\right]_{\mathrm{o}}$ than at $0.5 \mathrm{mM}\left[\mathrm{Ca}^{2+}\right]_{\mathrm{o}}$; therefore, we investigated the effects of PMA on capsaicin-induced currents in L3 and L5 PCs at $2 \mathrm{~mm}\left[\mathrm{Ca}^{2+}\right]_{\mathrm{o}}$.

In an L3 PC, the first puff application of capsaicin in the presence of $10 \mu \mathrm{M}$ PMA induced a large $I_{\text {cap }}$, concomitantly enhancing the current response to the ramp pulse (Fig. $4 A a$ ). The $I_{\text {cap }}$ displayed little decay even after the offset of the first puff application (Fig. 4Aa). The subsequent puff applications of capsaicin in the presence of PMA induced little inward current $\left({ }^{\$} p>0.3\right)$ and hardly increased the conductances $\left({ }^{\$} p>0.7\right)$ (Fig. $4 A a-A d)$. These results suggest that, in L3 PCs, both the $I_{\text {cap }}$ and $G_{\text {cap }}$ attained maximum plateau levels in response to the first puff application of capsaicin in the presence of PMA, most likely due to the strong enhancement of $I_{\text {cap }}$ by PKC activation. Similarly, in L5 PCs, the respective puff applications of capsaicin in the presence of PMA induced the respective $I_{\text {cap }}$ with little desensitization, which were summated to attain a maximal plateau level (Fig. $4 B$ ). Subsequently, in L5 PCs, PMA appeared to alleviate the desensitization of $I_{\text {cap }}$, which resulted in the conversion of tachyphylaxis into facilitatory $\left(I_{\text {cap }},{ }^{\$} p<0.002 ; G_{\text {cap }},{ }^{\$} p<0.04\right)$ summation (compare Figs. $4 B c, B d, 3 B c, B d$ ). Thus, PKC activation 
A

a

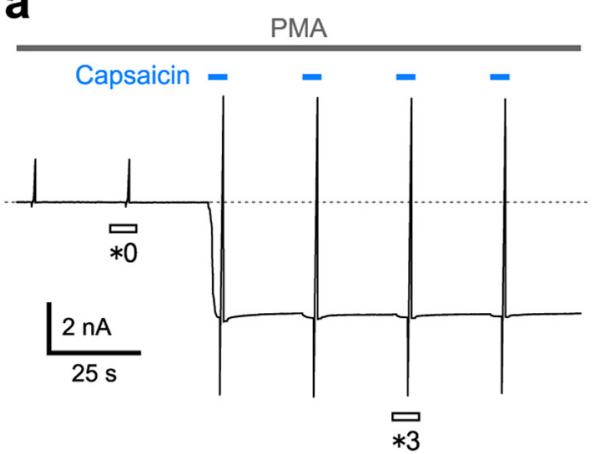

b

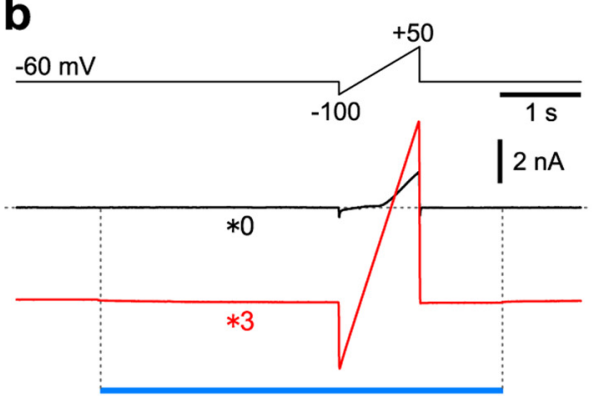

$20 \mu \mathrm{M}$ Capsaicin

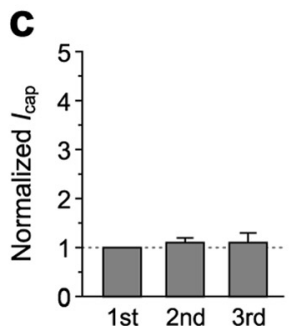

d

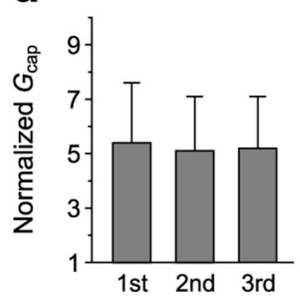

B
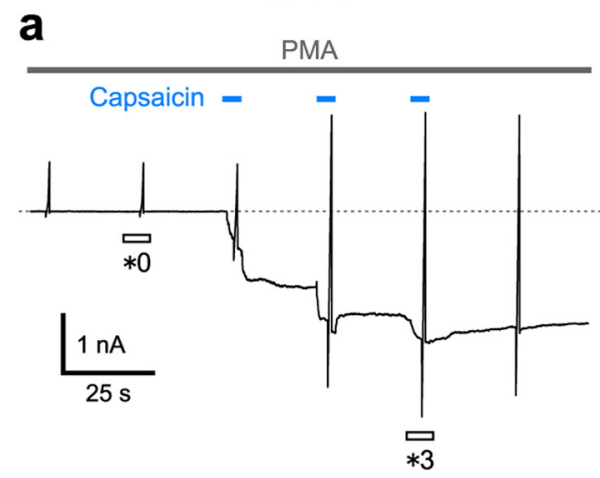

b

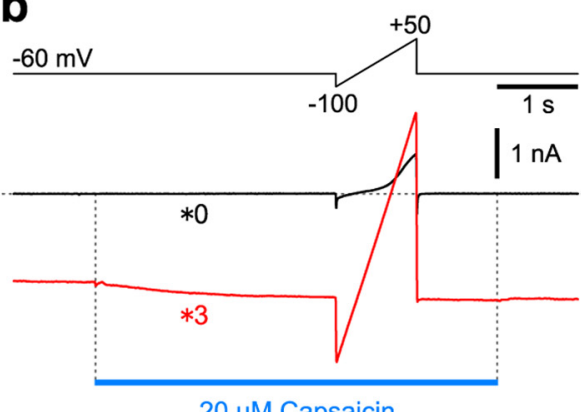

20 M Capsaicin

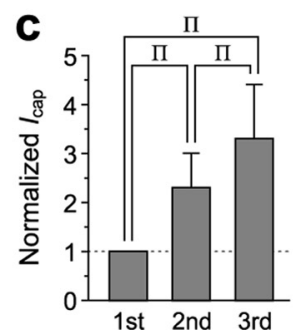

d

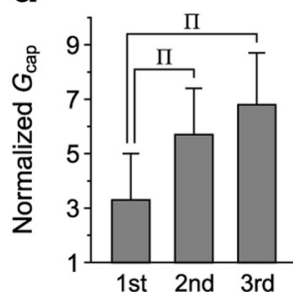

Figure 4. Differential enhancement of capsaicin-induced currents by PKC activation in L3 and L5 PCs in Gu-I. Aa, Ba, Sample traces of continuous recordings of cap $_{\text {cap }}$ induced by $5 \mathrm{~s}$ puff application of $20 \mu \mathrm{m}$ capsaicin and the current responses to the ramp pulses ( $\boldsymbol{A} \boldsymbol{b}, \boldsymbol{B} \boldsymbol{b}$, top) applied before and during respective $5 \mathrm{~s}$ puff applications of $20 \mu \mathrm{m}$ capsaicin (blue bars), in the presence of $10 \mu \mathrm{m}$ PMA in L3 $(\boldsymbol{A})$ and $\mathrm{L} 5(\boldsymbol{B}) \mathrm{PCS}$ at $2 \mathrm{~mm}\left[\mathrm{Ca}^{2+}\right]_{0}$. Holding potential, $-60 \mathrm{mV}$. $\boldsymbol{A} \boldsymbol{b}, \boldsymbol{B} \boldsymbol{b}$, Top, Positive ramp pulse from -100 to $+50 \mathrm{mV}$ for $1 \mathrm{~s}$ applied at a holding potential of $-60 \mathrm{mV}$. Bottom, Superimposed enlarged traces of current responses seen during the respective time periods indicated with open horizontal bars in $\boldsymbol{A a}\left({ }^{*} 0\right.$ and $\left.{ }^{*} 3\right)$ and $\boldsymbol{B a}\left({ }^{*} 0\right.$ and $\left.{ }^{*} 3\right)$. The blue bars indicate the duration and timing of puff applications of capsaicin. $A c, B c$, Mean ( $+S D)$ amplitudes of $I_{\text {cap }}$ obtained in response to the first through the third puff application of capsaicin, normalized to that obtained during the first puff application in $\mathrm{L} 3$ (second, $1.1 \pm 0.1$; third, $1.1 \pm 0.2 ; n=5)(\boldsymbol{A c})$ and $\mathrm{L} 5 \mathrm{PCS}$ (second, $2.3 \pm 0.7$; third, $3.3 \pm 1.1 ; n=5)(\boldsymbol{B C}) .{ }^{\Pi} p<0.05 . \boldsymbol{A d}, \boldsymbol{B d}$, Mean $(+S \mathrm{~S})$ values of $G_{\text {cap }}$ obtained in response to the first through the third puff application of capsaicin, normalized to that obtained in the control conditions in L3 (first, $5.4 \pm 2.2 ;$ second, $5.1 \pm 2.0$; third, $5.2 \pm$ $1.9 ; n=5)(\boldsymbol{A d})$ and L5 PCs (first, $3.3 \pm 1.7$; second, $5.7 \pm 1.7$; third, $6.8 \pm 1.9 ; n=5)(\boldsymbol{B d}) .{ }^{\Pi} p<0.05$.

changed the TRPV1 response in L5 PCs (Fig. $4 B c, B d$ ) to be similar to that in L3 PCs in the absence of PMA (Fig. 3Ac,Ad).

\section{Effects of capsaicin on columnwise excitation in Gu-I}

Using optical imaging, we next examined whether the activation of TRPV1 in L3 and L5 PCs of Gu-I could induce the coordination of neural activity between Gu-I and Au-I in the tiltedhorizontal slice preparation (see Materials and Methods). Following stimulation with $4 \mu \mathrm{A}\left(=1.2 \times \mathrm{Th}^{\star}\right)$ (see Materials and Methods) applied to a site $200 \mu \mathrm{m}$ rostral to MCA in L4 of Gu-I (Fig. 5Ca), an initial optical response was evoked in L4 with a latency of $1 \mathrm{~ms}$ (Fig. 5Aa, arrowhead), which subsequently spread bidirectionally into L2/3 and L5, displaying a columnar spatial pattern both in the absence and presence of capsaicin (Fig. $5 A a, B a, 5-16 \mathrm{~ms})$ in 8 of the 13 slices examined (62\%). This columnar excitation slowly faded out in the absence of capsaicin (Fig. 5Aa), whereas in the presence of capsaicin, it was followed by a second and third excitation spread extending toward the caudal end of the imaged area of the insular cortex (Fig. $5 \mathrm{Ba}$, 103-399 ms), in five of the eight slices.

By referring to the apparent boundary of the optical responses at 5-16 ms after L4 stimulation (Fig. 5Aa), we expediently divided the imaged area into four columns (columns $1-4$; Fig. $5 \mathrm{Cb}$, vertical dotted lines; see Discussion). Columns 1-3 were located in the DI region ( $\mathrm{Gu}-\mathrm{I})$, and most of column 4 was located in the GI/DI region (Au-I) (Fig. $5 \mathrm{Ca}, \mathrm{Cb}$; also see Fig. $1 C$ ). The optical responses evoked at the regions of interest (ROIs) (Fig. $5 \mathrm{Cb}$ ) in L3 and L 5 in columns $1-3$ by L4 stimulation in column 2 showed rapid increases in amplitude with slow and monotonic decay, while those in column 4 displayed no rapid increase (Fig. $5 A b, A c)$. The time-to-peak and the half-duration of the optical responses in L3 in the stimulated column 2 were $13 \pm 1$ and $91 \pm 21 \mathrm{~ms}$, respectively $(n=5$; Fig. $5 A b, A c)$. In the presence of capsaicin, however, the initial responses in columns $1-3$ were followed by a second and third wave, whereas such waves observed in column 4 were not preceded by any initial sharp com- 
A

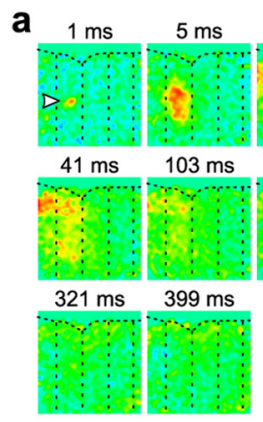

b

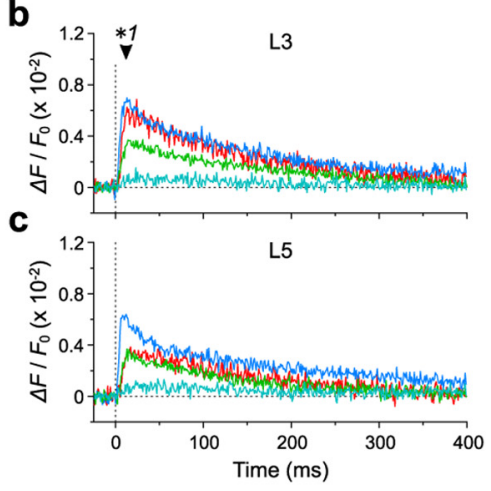

B
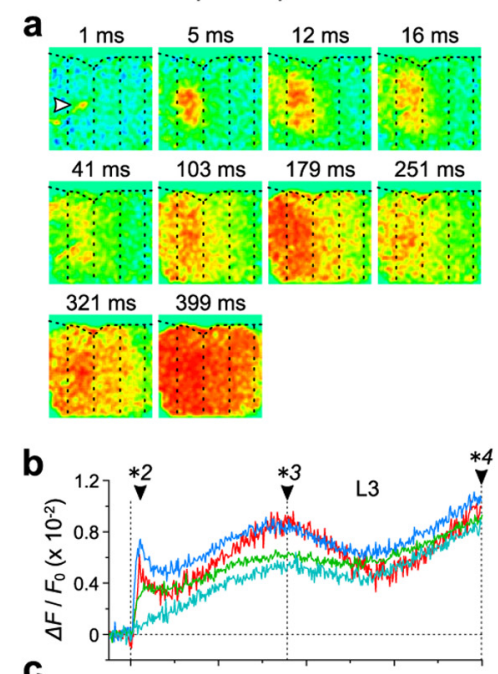

C

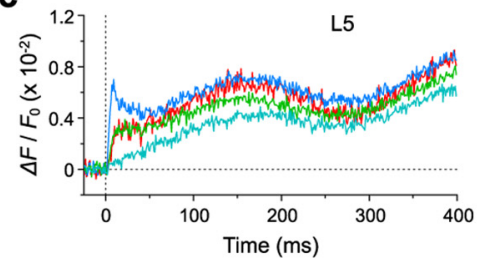

C
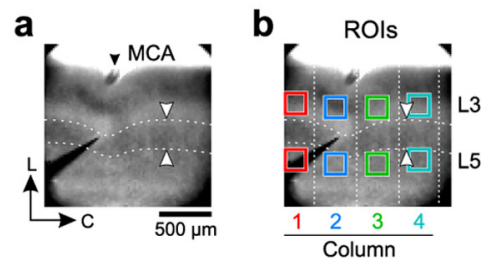

D

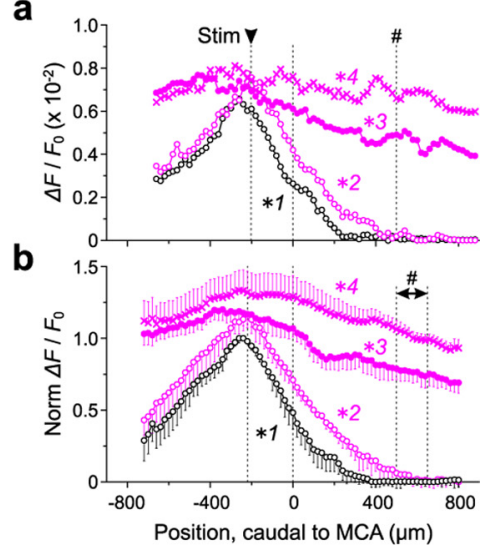

Figure 5. Oscillatory synchronization between $\mathrm{Gu}$-I and $\mathrm{Au}$-l induced by $\mathrm{L} 4$ stimulation in the presence of capsaicin. $\mathbf{A a}, \mathbf{B a}$, Sample pseudocolor images of optical responses induced by $4 \mu \mathrm{A}$ $\left(=1.2 \times \mathrm{Th}^{*}\right) \mathrm{L} 4$ stimulation in the absence $(\mathbf{A a})$ and presence $(\mathbf{B a})$ of $10 \mu \mathrm{m}$ capsaicin. The arrowheads indicate the initial optical responses evoked in $\mathrm{L} 4 \mathrm{\text {with }}$ a latency of $1 \mathrm{~ms}$. The vertical dotted lines showing the presumed column boundaries: The four columns were assigned numbers $1-4$ from left to right ( $\boldsymbol{C} b)$. $\boldsymbol{A} \boldsymbol{b}, \boldsymbol{A c}, \boldsymbol{B} \boldsymbol{b}, \boldsymbol{B} \boldsymbol{c}$, Temporal profiles at R0ls in $\mathrm{L} 3(\boldsymbol{b})$ and $\mathrm{L} 5$ ( $\boldsymbol{c}$ ) in columns $1-4$ represented by red, blue, green, and cyan traces, respectively, in the absence $(\boldsymbol{A})$ and presence $(\boldsymbol{B})$ of capsaicin. The filled arrowheads indicate the peak time points of the initial responses $\left({ }^{*} 1\right.$ and ${ }^{*} 2$ ) and the second wave (*3) and the end point of the recording time window (*4). C, Bright-field images of Gu-I. Stimulating electrode placed at $200 \mu m$ rostral to MCA (filled arrowhead) in L4 in $\mathrm{Gu}-\mathrm{I}(\boldsymbol{a})$. A pair of facing open arrowheads indicates the boundary between $\mathrm{Gu}-\mathrm{I}(\mathrm{DI})$ and $\mathrm{Au}-\mathrm{I}(\mathrm{GI} / \mathrm{DI})$. The vertical dotted lines indicate presumed column boundaries ( $\boldsymbol{b}$ ). The colored squares placed in $\mathrm{L} 3$ and $\mathrm{L} 5$ in columns $1-4$ represent ROIs $(\boldsymbol{b})$. The traces representing temporal profiles in columns $1-4(\boldsymbol{A} \boldsymbol{b}, \boldsymbol{A c}, \boldsymbol{B} \boldsymbol{b}, \boldsymbol{B} \boldsymbol{c})$ are colored in accordance with the colors of ROIs in columns $1-4$. L, Lateral; C, caudal. Da, Horizontal spatial profiles of the optical response in $\mathrm{L} 3$ at the time point of ${ }^{*} 1$ (black circles) shown in $\boldsymbol{A b}$ and at the time points of ${ }^{*} 2$ (purple open circles), ${ }^{*} 3$ (purple filled circles), and ${ }^{*} 4$ (purple crosses) shown in $\boldsymbol{B} \boldsymbol{b}$. The pound sign (\#) indicates the position of the border between Gu-I and Au-I. Db, Averaged ( \pm SD) horizontal spatial profiles ( $n=5$ ) in L3 at the peak time points of the initial sharp responses in the absence and presence of capsaicin (black and purple open circles, respectively), and at the peak time point of the second wave and the end point of the recording time window in the presence of capsaicin (purple filled circles and crosses, respectively). The pound sign (\#) indicates the positions of the borders between Gu-I and Au-I ranging from 500 to $650 \mu \mathrm{m}$ caudal to MCA.

ponent (Fig. $5 B b, B c$ ). The time-to-peak of the initial response and the peak time of the second wave of the temporal profiles in L3 in the stimulated column 2 were $11 \pm 1$ and $160 \pm 12 \mathrm{~ms}$, respectively $(n=5)$.

As seen in the horizontal spatial profiles of the optical responses in L3 (Fig. $5 D a, D b$ ) at the peak time points of the initial responses in the stimulated column in the absence (12 ms; Fig. 5Ab, $\left.{ }^{\star} 1\right)$ and presence ( $11 \mathrm{~ms}$; Fig. $5 B b,{ }^{\star} 2$ ) of capsaicin, the amplitudes of the initial optical responses declined to almost zero as the measuring point was moved toward the region of Au-I (black and purple open circles). However, at the peak time point of the second wave $(179 \mathrm{~ms}$, ${ }^{\star} 3$ ) and at the end of the recording time window (399 ms, ${ }^{*} 4$ ) (Fig. $5 B b$ ), the amplitudes of the optical responses at the caudal end of column 4 remained considerable (Fig. 5Da, Db, purple filled circles and crosses). Thus, the excitation initiated in column 2 of Gu-I by L4 stimulation propagated into more rostral and caudal columns with repetition of the excitation surges, leading to an oscillation-like synchronization across the entire Gu-I (columns 1-3) and at least the rostral portion of Au-I (column 4).

\section{Capsaicin-induced oscillation leads to synchronization between $\mathrm{Gu}-\mathrm{I}$ and $\mathrm{Au}-\mathrm{I}$}

We next examined whether stronger stimulation $(6 \mu \mathrm{A}=1.8 \times$ $\left.\mathrm{Th}^{\star}\right)$ could induce a direct excitation spread from $\mathrm{Gu}-\mathrm{I}$ to $\mathrm{Au}-\mathrm{I}$ in the absence of capsaicin. In response to $6 \mu \mathrm{A}$ stimulation, a larger optical response was induced (5-11 ms) both in the absence and presence of capsaicin (Fig. $6 \mathrm{Aa}, \mathrm{Ba}$ ) in 7 of the 12 slices examined (58\%) compared with the columnar pattern evoked by $1.2 \times \mathrm{Th}^{\star}$ stimulation (Fig. 5Aa,Ba, 5-12 ms). The larger initial optical response rapidly faded out in the absence of capsaicin (Fig. $6 \mathrm{Aa}, 27$ $\mathrm{ms}$ ), whereas in the presence of capsaicin, it was followed by a second and third excitation spread extending toward the caudal end of the imaged area of the insular cortex (Fig. 6 Ba, 140-400 $\mathrm{ms}$ ) in five of the seven slices. Given that the columnar width would not markedly vary among slices, we expediently divided the imaged area into four columns (Fig. $6 A a, B a$, vertical dotted lines). As revealed in the temporal profiles (Fig. 6Ab,Bb), sharply increasing responses were observed in columns 1-3 of Gu-I, whereas a much smaller delayed response was observed in column 4 . The initial response obtained with $1.8 \times \mathrm{Th}^{\star}$ stimulation decayed much more rapidly compared with those obtained with $1.2 \times \mathrm{Th}^{\star}$ stimulation (Fig. $5 A b$ ), as revealed by a significant $\left({ }^{\dagger} p<0.001\right)$ difference in half-duration $\left(1.8 \times \mathrm{Th}^{\star} \mathrm{vs} 1.2 \times \mathrm{Th}^{*}\right.$; $24 \pm 11 \mathrm{~ms}, n=5$, vs $91 \pm 21 \mathrm{~ms}, n=5)$. This finding suggests that the excitation induced by the stronger stimulation might have been immediately followed by inhibition; thus, the direct excitation spread from $\mathrm{Gu}-\mathrm{I}$ to $\mathrm{Au}-\mathrm{I}$ would be suppressed despite the application of stronger stimulation. 
A

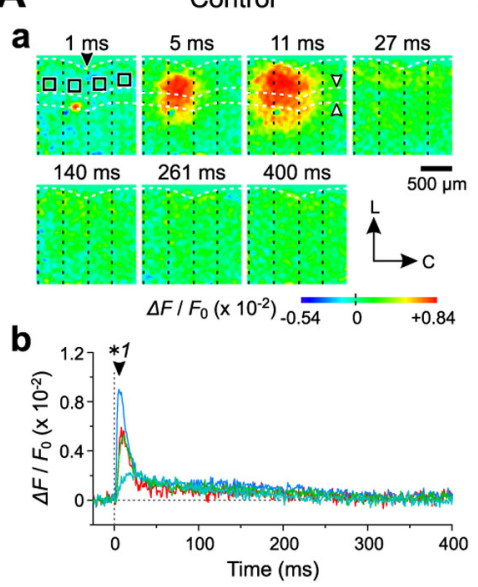

$D_{a}$

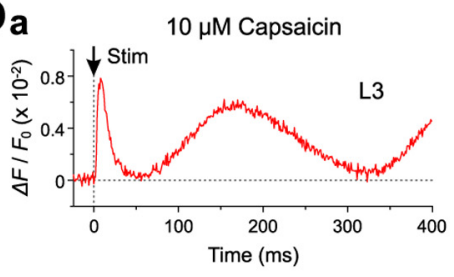

E

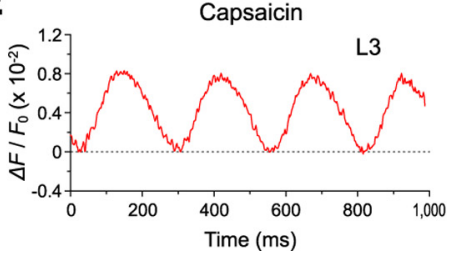

B
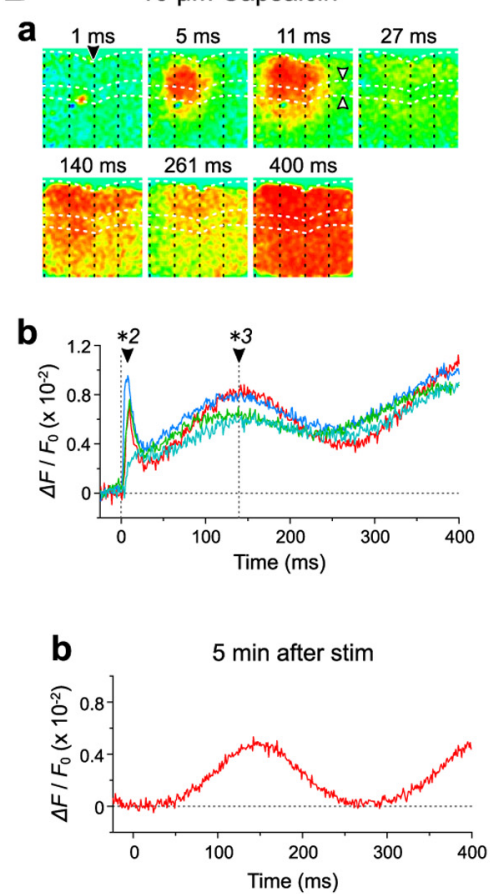

$F_{\mathbf{a}}$

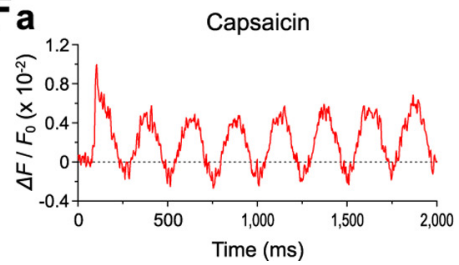

C

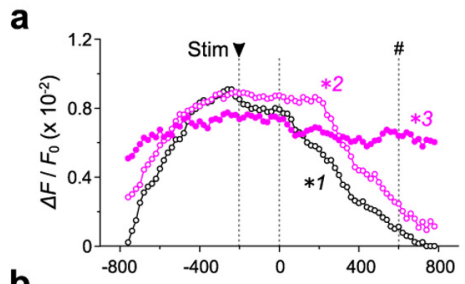

b
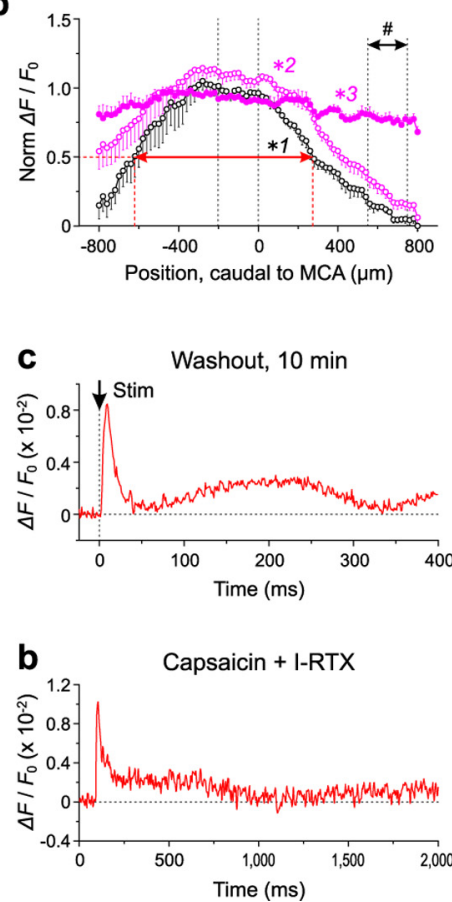

Figure 6. Facilitation of oscillatory synchronization between $\mathrm{Gu}$-I and Au-I by stronger $L 4$ stimulation. $\boldsymbol{A} \boldsymbol{a}, \boldsymbol{B} \boldsymbol{a}$, Sample pseudocolor images of optical responses induced in the absence $(\boldsymbol{A})$ and presence $(\boldsymbol{B})$ of $10 \mu \mathrm{m}$ capsaicin by $6 \mu \mathrm{A}\left(=1.8 \times \mathrm{Th}^{*}\right) \mathrm{L} 4$ stimulation applied to a site $200 \mu \mathrm{m}$ rostral to MCA (filled arrowheads) in L4 of Gu-I in a slice with the boundary (open arrowheads) between Gu-I (DI region) and Au-I (GI/DI region) at $600 \mu \mathrm{m}$ caudal to MCA. The vertical dotted lines showing the presumed column boundaries. The four columns were assigned numbers $1-4$ from left to right. The black squares represent ROIs in columns $1-4$. L, Lateral; $C$, caudal. $\boldsymbol{A} \boldsymbol{b}, \boldsymbol{B} \boldsymbol{b}$, Temporal profiles at L3 ROIs in columns $1-4$ represented by red, blue, green, and cyan traces, respectively, in the absence $(\boldsymbol{A} \boldsymbol{b})$ and presence $(\boldsymbol{B} \boldsymbol{b})$ of capsaicin. The filled arrowheads indicate the peak time points of the initial responses $\left({ }^{*} 1\right.$ and $\left.{ }^{*} 2\right)$ and the second wave $\left({ }^{*} 3\right)$. Ca, Horizontal spatial profile of the optical response in $\mathrm{L} 3$ at the time point of ${ }^{*} 1$ (black circles) shown in $\boldsymbol{A} \boldsymbol{b}$ and at the time points of ${ }^{*} 2$ (purple open circles) and ${ }^{*} 3$ (purple filled circles) shown in $\boldsymbol{B} \boldsymbol{b}$. The pound sign (\#) indicates the position of the border between Gu-l and Au-I. $(b$, Averaged $( \pm S D)$ horizontal profiles $(n=5)$ at the peak time points of the initial sharp response in the absence and presence of capsaicin (black and purple open circles, respectively) and at the peak time points of the second waves in the presence of capsaicin (purple filled circles). The pound sign (\#) indicates the positions of the borders between Gu-I and Au-I ranging from 550 to $750 \mu \mathrm{m}$ caudal to MCA. Red left-right arrow indicates the half-width of the horizontal profile. $\boldsymbol{D}$, A representative example of an initial sharp response followed by a slow oscillation induced in the presence of $10 \mu \mathrm{m}$ capsaicin by $6 \mu \mathrm{AL}$ L stimulation (a). Persistence of the oscillation 5 min after single stimulation (b). Marked diminution of the slow oscillation following the unaltered initial response $10 \mathrm{~min}$ after capsaicin washout $(\boldsymbol{c})$. $\boldsymbol{E}$, Once triggered, the oscillation persisted as long as capsaicin $(5 \mu \mathrm{M})$ was present. $\boldsymbol{F}, \mathrm{I}-\mathrm{RTX}(0.5 \mu \mathrm{M})$ addition to the perfusate including capsaicin $(10 \mu \mathrm{M})$ abolished the capsaicin-induced oscillatory response, leaving the initial response unaffected $(\boldsymbol{a}, \boldsymbol{b})$.

Indeed, as revealed in the horizontal spatial profiles in L3 (Fig. $6 \mathrm{Ca}, \mathrm{Cb},{ }^{\star} 1$, black circles) at the peak time point of the initial sharp response $\left({ }^{\star} 1 ; 10 \pm 1 \mathrm{~ms} ; n=5\right.$; Fig. $\left.6 A b\right)$, the amplitude that peaked at the center of column 2 declined to almost zero toward the caudal end of column 4, despite the significantly larger $\left({ }^{\dagger} p<0.005\right)$ half-width $(860 \pm 42 \mu \mathrm{m} ; n=5)$ of the horizontal spatial profile compared with that $(588 \pm 49 \mu \mathrm{m} ; n=$ 5 ) obtained with the weaker stimulation (Fig. $5 \mathrm{Db}$ ). Similarly, in the presence of capsaicin, the amplitude at the peak time point of the initial sharp response (Fig. $6 B b,{ }^{\star} 2$ ) declined markedly toward the caudal end of column 4 (Fig. $6 \mathrm{Ca},{ }^{\star} 2$ ). However, after the oscillation-like response was induced by the application of capsaicin, the amplitude of the optical responses at the peak time point of the second wave did not decline markedly even at the caudal end of column 4 (Fig. 6Ca, ${ }^{\star} 3$ ). The divergence in the amplitude of the second wave of oscillation-like responses among the four ROIs was considerably smaller compared with that in- duced with $1.2 \times \mathrm{Th}^{\star}$ stimulation (compare Figs. $5 B b, 6 B b$ ). This result was consistent with a significant $\left({ }^{\dagger} p<0.02\right)$ difference in the percentile decline of amplitude at $800 \mu \mathrm{m}$ caudal to MCA between the horizontal profiles of the second waves obtained with $1.2 \times \mathrm{Th}^{\star}(38 \pm 6 \% ; n=5$; Fig. $5 \mathrm{Db})$ and $1.8 \times \mathrm{Th}^{\star}$ intensities $(25 \pm 3 \%$; $n=5$; Fig. $6 C b)$. This finding suggests that a stronger stimulation could facilitate the capsaicin-induced excitation spread from $\mathrm{Gu}-\mathrm{I}$ to $\mathrm{Au}-\mathrm{I}$.

Once the oscillation-like response was induced by the presence of capsaicin following L4 stimulation (Fig. $6 \mathrm{Da}$ ), it persisted for at least $15 \mathrm{~min}$ in most cases $(n=8$ of 10 ; Fig. $6 \mathrm{Db}, E)$ and was markedly attenuated $10 \mathrm{~min}$ after washout of capsaicin (Fig. $6 D c)$. The time interval between the two given points that were located separately in the two rising phases of the optical responses and have an identical amplitude was almost constant in Figure 6, $D a$ and $D b$, and was the same between Figure 6, Da and $D b$. This notion suggests the presence of the periodicity of the optical re- 
$A_{\text {a }}$

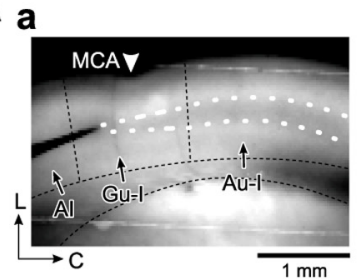

B

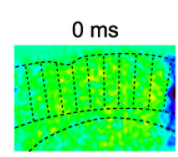

$128 \mathrm{~ms}$

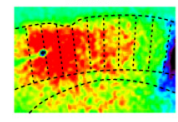

$376 \mathrm{~ms}$

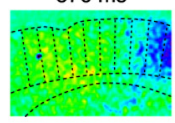

$552 \mathrm{~ms}$
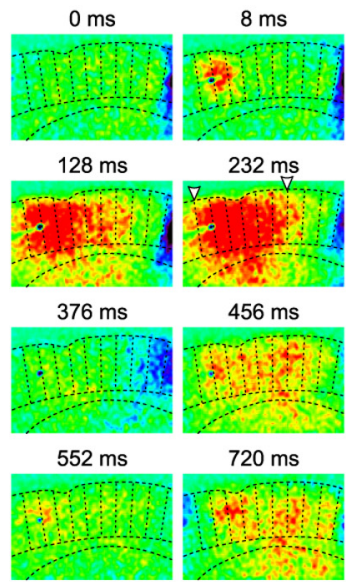

$232 \mathrm{~ms}$

$456 \mathrm{~ms}$

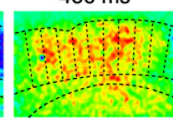

$720 \mathrm{~ms}$ b

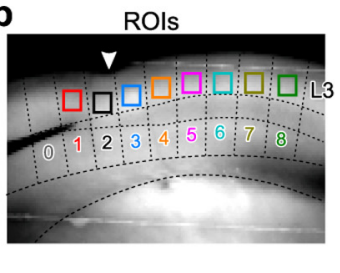

$10 \mu \mathrm{M}$ Capsaicin
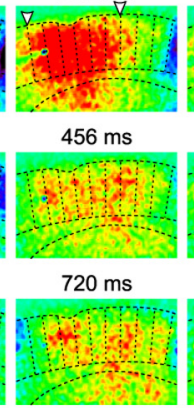

$16 \mathrm{~ms}$

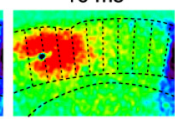

$272 \mathrm{~ms}$

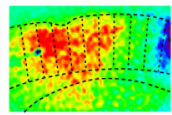

$472 \mathrm{~ms}$

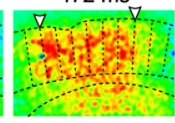

$800 \mathrm{~ms}$

$\Delta F / F_{0}\left(\times 10^{-2}\right)$

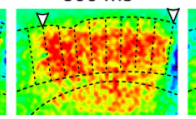

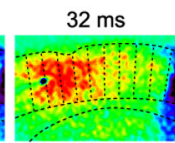

$320 \mathrm{~ms}$

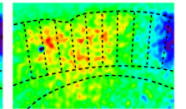

$520 \mathrm{~ms}$

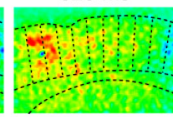

$1400 \mathrm{~ms}$

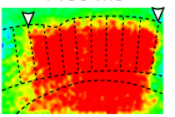

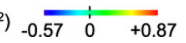

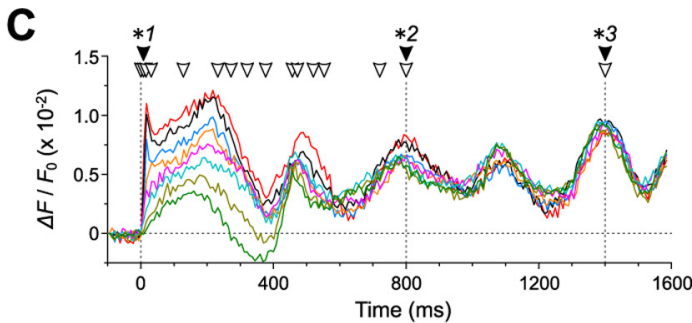

D
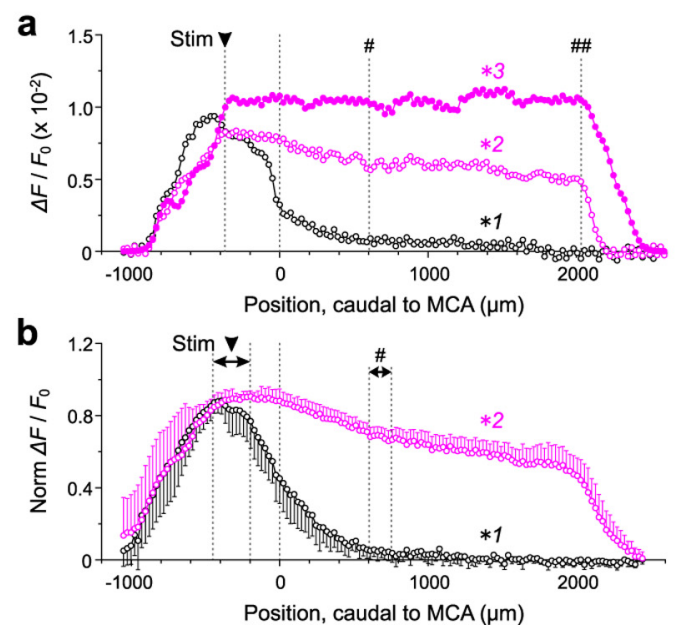

Figure 7. Synchronous oscillation between Gu-I and Au-I in the presence of capsaicin. $\boldsymbol{A}$, Resting-light-intensity images of the insular cortex in the tilted-horizontal slice. Stimulating electrode placed at $\mathrm{L} 4(400 \mu \mathrm{m}$ rostral to MCA) of $\mathrm{Gu}-\mathrm{I}(\boldsymbol{a})$. Al, agranular insula. The vertical dotted lines showing the presumed column boundaries $(\boldsymbol{b})$. The colored squares representing R0ls in $\mathrm{L} 3$ of $\mathrm{Gu}-\mathrm{I}$ (columns 1-3), Au-I (columns 5-8), and the Gu-I/Au-I boundary area (column 4). L, Lateral; C, caudal. B, Sample pseudocolor images of optical responses evoked by stimulation of Gu-I L4 at $6 \mu$ A $\left(=1.8 \times \mathrm{Th}^{*}\right)$ in the presence of $10 \mu \mathrm{m}$ capsaicin. The arrowheads indicate the rostral and caudal ends of columnwise excitation spread. $\boldsymbol{C}$, Traces representing temporal profiles at respective ROls in columns 1-8 in the presence of capsaicin are colored in accordance with respective colors of ROls in columns 1-8. The open arrowheads indicate the time points at which the respective pseudocolor-images $(\boldsymbol{B})$ were obtained. The filled arrowheads indicate the peak time points of the initial responses $\left({ }^{*} 1\right)$ and the fourth $\left({ }^{*} 2\right)$ and sixth $\left({ }^{*} 3\right)$ waves. Da, Horizontal spatial profiles of the optical response in L3 at the time points of *1 (black circles), ${ }^{*} 2$ (purple open circles), and *3 (purple filled circles) shown in C. The pound sign (\#) indicates the position of the border between Gu-I and Au-I. The double pound sign (\#\#) indicates the position of the caudal end of Au-I. Db, Averaged ( \pm SD) horizontal profiles $(n=5)$ at 8 ms and the peak time point of the fourth wave (black and purple circles, respectively). The arrowhead and the pound sign (\#) indicate the range of the positions of the stimulation sites and that of the positions of the borders between Gu-I and Au-I ranging from 600 to $750 \mu \mathrm{m}$ caudal to MCA, respectively.

sponses. The mean periodicity was at $3.8 \pm 0.1 \mathrm{~Hz}(n=8)$. In the five slices examined, an addition of I-RTX $(0.5 \mu \mathrm{M})$ to the perfusate containing capsaicin $(10 \mu \mathrm{M})$ consistently abolished the capsaicin-induced oscillatory response, leaving the initial response unaffected (Fig. $6 F a, F b$ ). Thus, the oscillatory synchronization between $\mathrm{Gu}-\mathrm{I}$ and $\mathrm{Au}-\mathrm{I}$ was induced through the activation of TRPV1.

\section{Capsaicin induces oscillatory network synchronization between $\mathrm{Gu}-\mathrm{I}$ and $\mathrm{Au}-\mathrm{I}$}

We further examined how far the oscillatory excitation initiated in $\mathrm{Gu}-\mathrm{I}$ propagates beyond the boundary between $\mathrm{Gu}-\mathrm{I}$ and $\mathrm{Au}-\mathrm{I}$ using a system that captures optical responses in Gu-I (DI region) and the entire Au-I (GI/DI region) (Fig. $1 \mathrm{Ca}$, the rectangular area labeled MC) at a sampling rate of $125 \mathrm{~Hz}$. Due to this limited sampling rate, the peak amplitude of the initial sharp response would be underestimated in these experiments.

Stimulation of L4 in Gu-I at $6 \mu \mathrm{A}\left(=1.8 \times \mathrm{Th}^{*}\right.$; Fig. $\left.7 \mathrm{Aa}\right)$ in the presence of $10 \mu \mathrm{M}$ capsaicin induced an initial optical response with a columnar spatial pattern in Gu-I at $8 \mathrm{~ms}$ (Fig. 7B). Thereafter, the oscillatory optical response extended over Gu-I and Au-I (Fig. 7B). A similar oscillatory excitation spread was observed in a total of five of the eight slices examined. By referring to the apparent boundaries of the respective optical responses in Gu-I (Fig. 7B), we divided the imaged area into nine columns (columns 0-8; Fig. 7Ab). Because the boundary between DI and GI/DI was located at $\sim 600 \mu \mathrm{m}$ caudal to MCA within column 4 (Fig. 7Aa), Gu-I was represented in columns 1-3 and Au-I was represented in columns 5-8 (Fig. 7Ab). The optical response initiated in Gu-I column 1 rapidly spread caudally to column 3 (8-16 ms) and slowly propagated to columns 4-6 (232 ms) (Fig. $7 B)$. This rapid spread within $\mathrm{Gu}-\mathrm{I}$ was reflected in the rapid rise to the initial peak of the temporal profiles at the ROIs in columns $1-3$, and the slow propagation into Au-I was reflected in the slow rising phases of those in columns $4-8$ (Fig. 7C). This slow rise of the optical responses in Au-I (columns 4-6) peaked at 200-230 ms, almost simultaneously with the second wave of the optical responses in Gu-I (columns 1-3) (Fig. 7C).

Following the decay of those optical responses, the synchronized oscillatory response emerged after $400 \mathrm{~ms}$ following stimulation (Fig. 7 B, C). During the first cycle of the oscillation $(<400$ $\mathrm{ms}$ ), the amplitude of the optical responses was highest in the stimulated column 1 (Fig. $7 C$, red trace) and was smaller at more distant ROIs (Fig. $7 \mathrm{~B}, \mathrm{C}$ ). However, as the oscillation progressed, the amplitude divergence in the optical responses among the columns gradually decreased (Fig. 7C), and the caudal end of the excited region shifted to the caudal end of Au-I (bregma -0.5 $\mathrm{mm} \approx 2.0 \mathrm{~mm}$ caudal to MCA). Thus, a more widespread synchronous oscillation was induced over Gu-I and Au-I (Fig. 7B, $800 \mathrm{~ms}$ ), which was more clearly observed in the wide-range 

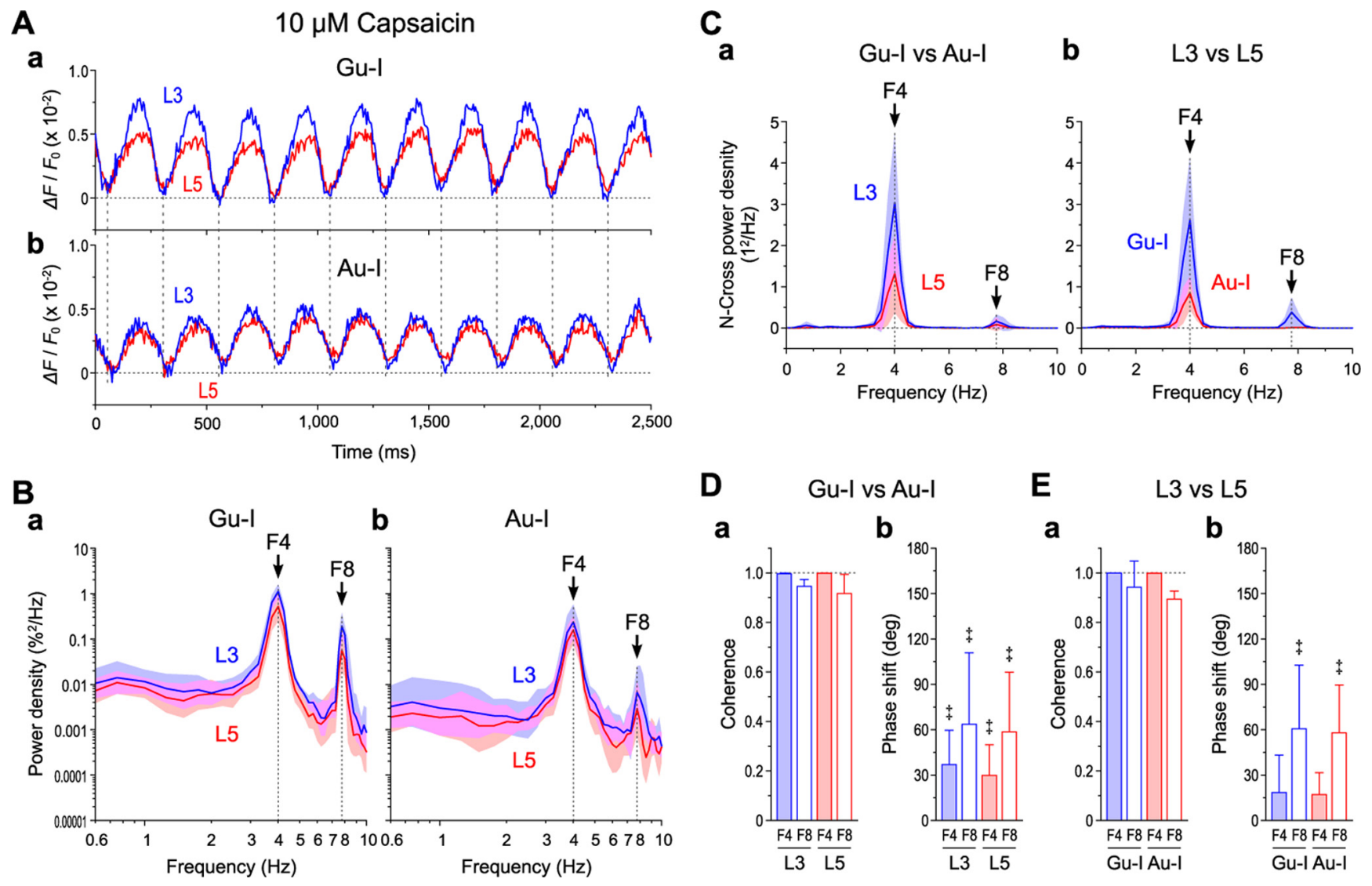

L3 vs L5

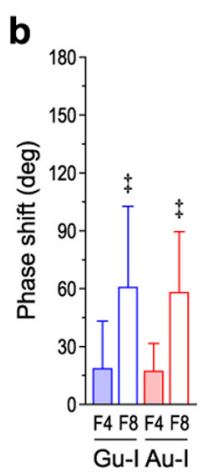

Figure 8. Theta synchronization between Gu-I and Au-I. $A$, Examples of temporal profiles of long-lasting oscillatory optical responses triggered by $6 \mu A(=1.8 \times$ Th*) Gu-I L4 stimulation in the presence of $5 \mu$ m capsaicin. Temporal profiles at ROls located in L3 (blue traces) and L5 (red traces) of Gu-I ( $\boldsymbol{a}$ ) and those of Au-I ( $\boldsymbol{b}$ ) were simultaneously obtained. The vertical dotted lines indicate

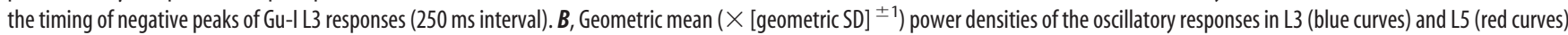
of Gu-I ( $(\boldsymbol{a})$ and those of Au-I $(\boldsymbol{b})(n=5)$. C, Mean ( \pm SD) cross-power densities between Gu-I and Au-l in L3 (blue curve) and L5 (red curve) (a) and those between L3 and L5 in Gu-I (blue curve) and Au-I (red curve) $(\boldsymbol{b})(n=5)$. $\boldsymbol{D}, \boldsymbol{E}$, Mean ( \pm SD) values of coherence $(\boldsymbol{a})$ and phase shift $(\boldsymbol{b})$ between $\mathrm{F} 4$ and F8 components of Gu-I and those of Au-I (D) and between those of $\mathrm{L} 3$ and those of $L 5$ ( $\boldsymbol{E}$ ) $(n=5)$. ${ }^{\ddagger} p<0.05\left(v s 0^{\circ}\right)$. Coherence between Gu-I and Au-I: L3 F4, $0.998 \pm 0.003 ; \mathrm{L} 3$ F8, $0.946 \pm 0.026 ; \mathrm{L} 5 \mathrm{~F} 4,0.998 \pm 0.001 ; \mathrm{L} 5 \mathrm{F8}, 0.916 \pm 0.078$. Coherence between L3 and L5: Gu-I F4, $0.999 \pm 0.001 ; \mathrm{Gu}-\mathrm{IF} 8,0.941 \pm 0.107 ; \mathrm{Au}-\mathrm{IF} 4,0.998 \pm 0.002 ; \mathrm{Au}-\mathrm{IF} 8,0.894 \pm 0.032$. Phase shift between Gu-I and Au-I: L3 F4, $37 \pm 23^{\circ} ; \mathrm{L} 3 \mathrm{~F} 8,64 \pm 47^{\circ} ; \mathrm{L} 5 \mathrm{~F} 4,30 \pm 20^{\circ} ; \mathrm{L} 5 \mathrm{~F} 8,59 \pm 39^{\circ}$. Phase shift between L3 and L5: Gu-IF4, $19 \pm 25^{\circ} ; \mathrm{Gu}-\mathrm{IF} 8,61 \pm 42^{\circ} ; \mathrm{Au}-\mathrm{IF} 4,17 \pm 14^{\circ} ; \mathrm{Au}-\mathrm{IF8}, 58 \pm 32^{\circ}$.

plateau-like horizontal profile of the optical response (Fig. $7 \mathrm{Da}$, ${ }^{\star} 2$ ) at the peak time point of the fourth wave (Fig. $7 C,{ }^{\star} 2$ ). The fourth wave was the earliest cycle required for the optical response to reach the caudal end of Au-I (Fig. 7Da, \#\#), as shown in the averaged horizontal spatial profile (Fig. 7Db) obtained at the peak time point of the fourth wave (peak time, $812 \pm 55 \mathrm{~ms} ; n=$ 5). Together with the results shown in Figures 5 and 6, it is likely that TRPV1-assisted excitation in Gu-I initiates the oscillatory coordination of neural activity between $\mathrm{Gu}-\mathrm{I}$ and Au-I, suggesting the presence of functional integration between Gu-I and $\mathrm{Au}-\mathrm{I}$.

\section{Theta-band synchronization between Gu-I and Au-I}

The wave of capsaicin-induced oscillatory optical responses that persisted for a long time ( $\geq 15 \mathrm{~min}$ ) after Gu-I L4 stimulation (Fig. 8A) (see also Figs. 6E, F, 7C) appeared not to be a simple sine wave. In each cycle of the waves in $\mathrm{Gu}-\mathrm{I}$ and $\mathrm{Au}-\mathrm{I}$, the onset of a positive wave in $\mathrm{Gu}-\mathrm{I}$ preceded that in $\mathrm{Au}-\mathrm{I}$ (vertical interrupted lines), indicating an excitation spread from $\mathrm{Gu}-\mathrm{I}$ to Au-I. The power spectral analysis of temporal profiles of the oscillatory optical responses in $\mathrm{Gu}-\mathrm{I}$ and $\mathrm{Au}-\mathrm{I}(n=5)$ revealed that the oscillation was mainly composed of two prominent frequency components of $3.5-4.5 \mathrm{~Hz}$ (F4) and 7-9 Hz (F8) (Fig. $8 B a, B b)$. Both $\mathrm{F} 4$ and $\mathrm{F} 8$ powers were significantly larger $\left({ }^{\ddagger} p<0.05\right)$ in L3 than in L5 in Gu-I but not in Au-I ( $\left.{ }^{\ddagger} p>0.3\right)$, while both F4 and F8 powers were also larger $\left({ }^{\ddagger} p<0.02\right)$ in $\mathrm{Gu}-\mathrm{I}$ than in Au-I.

As oscillatory synchronization between $\mathrm{Gu}-\mathrm{I}$ and $\mathrm{Au}-\mathrm{I}$ was apparent (Figs. 5-7), we performed a cross-power spectral analysis to evaluate the degree of synchronization between Gu-I and $\mathrm{Au}-\mathrm{I}$. The cross-power densities (Sklar et al., 1972) between Gu-I and Au-I in both L3 $\left({ }^{\ddagger} p<0.03\right)$ and L5 $\left({ }^{\ddagger} p<0.05\right)$ were prominent at $4 \mathrm{~Hz}(n=5$; Fig. $8 \mathrm{Ca})$, as were the cross-power densities between L3 and L5 in both Gu-I $\left({ }^{\ddagger} p<0.03\right)$ and Au-I $\left({ }^{\ddagger} p<0.02\right)$ $(n=5$; Fig. $8 \mathrm{Cb}$ ). Consistent with these observations, the coherence (Sklar et al., 1972) between Gu-I and Au-I in both L3 and L5 was very high $(\approx 1)$ at $4 \mathrm{~Hz}$ but slightly lower $(\approx 0.9)$ at $8 \mathrm{~Hz}(n=$ 5; Fig. $8 D a$ ), as was the coherence between L3 and L5 in both $\mathrm{Gu}-\mathrm{I}$ and $\mathrm{Au}-\mathrm{I}(n=5$; Fig. $8 \mathrm{Ea}$ ). There were significant positive phase shifts between F4 in Gu-I and that in Au-I in both L3 and L5 $\left({ }^{\ddagger} p<0.05\right)$, between F8 in Gu-I and that in Au-I in both L3 and L5 ( ${ }^{\ddagger} p<0.05$ ) (Fig. $8 \mathrm{Db}$ ), and between F8 in L3 and that in L5 in both Gu-I and Au-I ( $\left.{ }^{*} p<0.05\right)$ (Fig. 8 Eb). A smaller and insignificant $\left({ }^{\ddagger} p>0.08\right)$ shift was observed between F4 in L3 and that in L5 in both Gu-I and Au-I (Fig. 8 Eb). Thus, TRPV1 activation induced oscillatory synchronization between Gu-I and Au-I at the theta range as reflected in F4 and F8 components, and each 
A

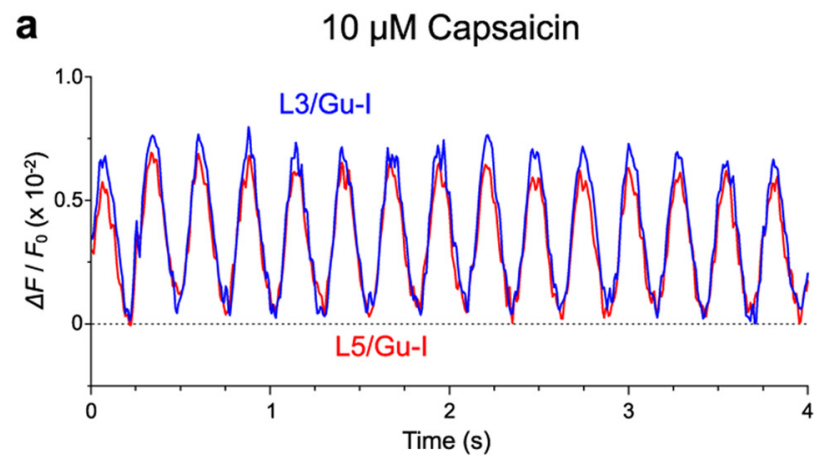

b

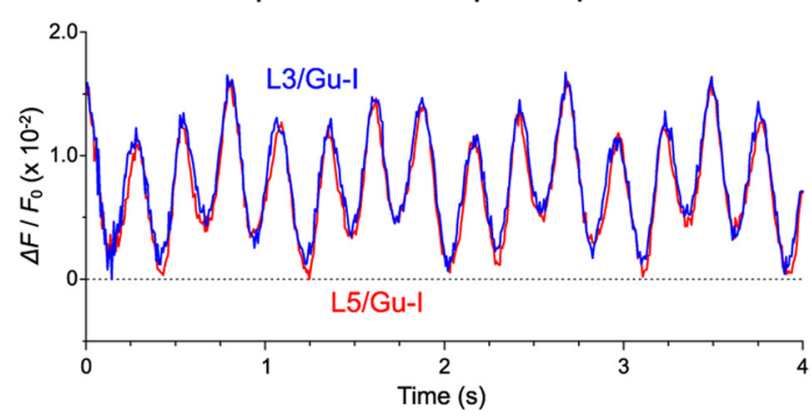

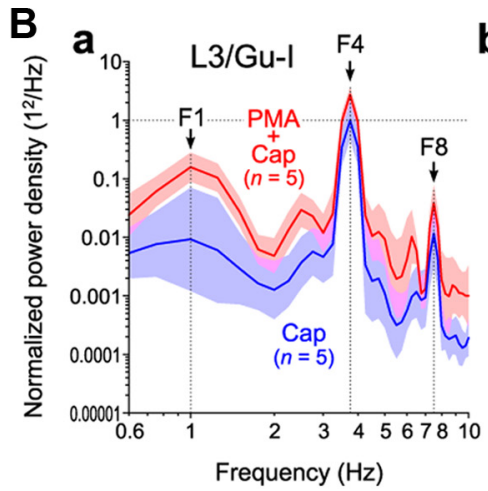

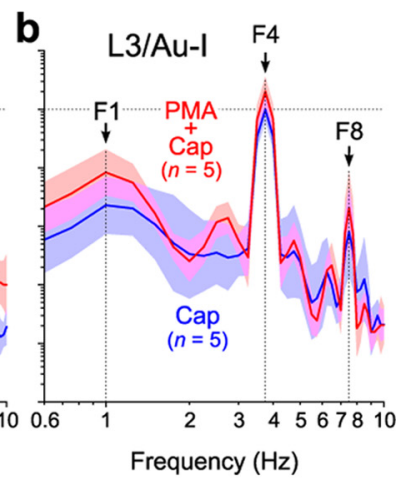

C

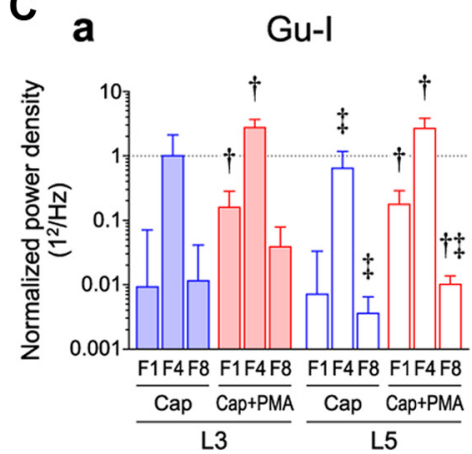

b

Au-I

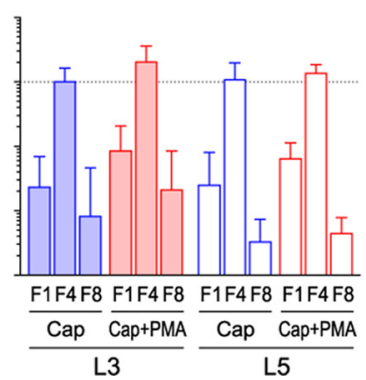

Figure 9. PMA-induced complex oscillations composed of 1 , 4 , and 8 Hz waves. $A$, A simple $(\boldsymbol{a})$ and a complex (b) oscillation induced by $4 \mu \mathrm{A}(=1.2 \times$ Th*) Gu-I L4 stimulation in L3 (blue traces) and $L 5$ (red traces) of the stimulated column in Gu-I of two different slice preparations after bath application of $10 \mu \mathrm{m}$ capsaicin alone and after bath application of $10 \mu \mathrm{m}$ capsaicin in the presence of $10 \mu \mathrm{M}$ PMA, respectively. $\boldsymbol{B}$, Geometric mean $\left(\times\right.$ [geometric SD] ${ }^{ \pm 1}$ ) power densities of the oscillatory responses in the presence of capsaicin without (blue curves) and with PMA (red curves) in L3 of Gu-I ( $n=5$ and 5 , respectively) (a) and Au-I ( $n=5$ and 5, respectively) (b). Optical responses in Gu-I and Au-I were obtained separately and nonpairwise. C, Geometric mean ( $\times$ [geometric $S D]^{ \pm 1}$ ) values of $\mathrm{F} 1, \mathrm{~F} 4$, and $\mathrm{F} 8$ power densities in $\mathrm{L} 3$ and $\mathrm{L} 5$ of $\mathrm{Gu}-\mathrm{I}(\boldsymbol{a})$ and $\mathrm{Au}-\mathrm{I}(\boldsymbol{b})$. The blue and red columns represent geometric mean power densities in the presence of capsaicin alone and with PMA, respectively. PMA significantly enhanced F1 and F4 components in L3 (solid columns) as well as in L5 (hollow columns) in Gu-I. ${ }^{\dagger} p<0.05$ (vs Cap); ${ }^{\ddagger} p<0.05$ (vs L5). Normalized values of the

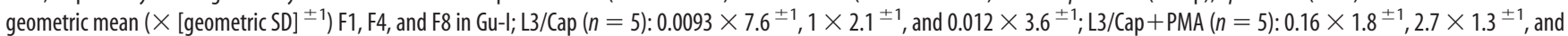
$0.038 \times 2.0^{ \pm 1} ; \mathrm{L} 5 / \mathrm{Cap}(n=5): 0.0072 \times 4.6^{ \pm 1}, 0.63 \times 1.9^{ \pm 1}$, and $0.0036 \times 1.8^{ \pm 1} ; \mathrm{L} 5 / \mathrm{Cap}+\mathrm{PMA}(n=5): 0.18 \times 4.6^{ \pm 1}, 2.6 \times 1.4^{ \pm 1}$, and $0.010 \times 1.3^{ \pm 1}$. Normalized values of the geometric mean $\left(\times\left[\right.\right.$ geometric SD] $\left.{ }^{ \pm 1}\right) \mathrm{F} 1, \mathrm{~F} 4$, and F8 in Au-l; L3/Cap $(n=5): 0.023 \times 3.0^{ \pm 1}, 1 \times 1.6^{ \pm 1}$, and $0.0081 \times 5.8^{ \pm 1} ; \mathrm{L} 3 / \mathrm{Cap}+\mathrm{PMA}(n=5): 0.084 \times 2.5^{ \pm 1}, 2.0 \times 1.8^{ \pm 1}$, and $0.021 \times 4.0^{ \pm 1} ;$ L5/Cap $(n=5): 0.025 \times 3.2^{ \pm 1}, 1.1 \times 1.8^{ \pm 1}$, and $0.0033 \times 2.2^{ \pm 1} ; L 5 /$ Cap + PMA $(n=5): 0.064 \times 1.8^{ \pm 1}, 1.4 \times 1.4^{ \pm 1}$, and $0.0044 \times 1.8^{ \pm 1}$.

cycle of such oscillatory responses might be initiated in the supragranular layer of Gu-I.

\section{PMA induced a complex oscillation composed of 1,4 , and 8 $\mathrm{Hz}$ waves}

Because the PKC activation similarly enhanced the two distinct $I_{\text {cap }}$ in L3 and L5 PCs (Fig. 4), we examined whether PMA modulates capsaicin-induced oscillations in Gu-I and Au-I. Optical responses in Gu-I and Au-I were obtained separately and nonpairwise. In the presence of $10 \mu \mathrm{M}$ capsaicin alone, Gu-I L4 stimulation consistently triggered apparently simple oscillations at $\sim 4 \mathrm{~Hz}$ in $\mathrm{Gu}-\mathrm{I}(0.2-0.4 \mathrm{~mm}$ rostral to MCA) (Fig. 9Aa; $n=5$ ), similar to the results shown in Figure 8. However, bath application of $10 \mu \mathrm{M}$ capsaicin in the presence of $10 \mu \mathrm{M}$ PMA induced complex oscillations (Fig. 9Ab). The power spectrum analysis (Fig. $9 B a, B b$, red curves) revealed that this complex oscillation was composed of the three frequency components of $3.5-4 \mathrm{~Hz}$ (F4), $7-8 \mathrm{~Hz}(\mathrm{~F} 8)$, and $0.7-1.5 \mathrm{~Hz}(\mathrm{~F} 1)$ in Gu-I $(n=5)$ and Au-I (1.5-2.0 mm caudal to MCA) $(n=5)$. To examine the effects of PMA on oscillation, all the power spectra obtained in the presence of capsaicin together with or without PMA were normalized to the geometric mean of the peak F4 obtained in the presence of capsaicin alone. In $\mathrm{Gu}-\mathrm{I}$, the geometric mean values of F1 and F4 power densities in L3 and L5 obtained in the presence of PMA and capsaicin $(n=5)$ were significantly larger $\left({ }^{\dagger} p<0.05\right)$ than those obtained in the presence of capsaicin alone $(n=5)$, while PMA significantly affected the F8 power densities in L5 $\left({ }^{\dagger} p<\right.$ 0.02 ) but not in $\mathrm{L} 3\left({ }^{\dagger} p>0.2\right.$ ) (Fig. $9 \mathrm{Ba}, \mathrm{Ca}$ ). In contrast, none of the geometric mean values of $\mathrm{F} 1, \mathrm{~F} 4$, and F8 power densities in $\mathrm{Au}-\mathrm{I}$ were significantly affected by PMA $\left({ }^{\dagger} p>0.05 ; n=5\right.$; Fig. $9 B b, C b)$. Such differential effects of PMA between oscillations in $\mathrm{Gu}-\mathrm{I}$ and $\mathrm{Au}-\mathrm{I}$ were consistent with the differential distribution of TRPV1 between Gu-I and Au-I (Fig. 2C). Collectively, these results showed that the oscillatory synchronization between $\mathrm{Gu}-\mathrm{I}$ and Au-I mainly at the theta range was induced by capsaicin regardless of the presence or absence of PMA.

\section{Capsaicin does not modulate spike trains induced by current pulse injections}

To understand the cellular mechanisms underlying the synchronized oscillations observed, we examined the effects of capsaicin on spike firings evoked in PCs of Gu-I. First, we characterized the firing properties of L3 and L5 PCs of Gu-I and examined the effects of $5 \mu \mathrm{M}$ capsaicin on spike trains induced by current pulse injections. In the absence of capsaicin, spike trains induced in L3 PCs by injection of current pulses displayed spike frequency adaptation (Fig. 10 Aa), whereas those induced in L5 PCs displayed little spike frequency adaptation (Fig. $10 \mathrm{Ba}$ ), as revealed by the changes in interspike intervals (ISIs) during spike trains (Fig. $10 A b, B b)$. Spike frequency adaptation could also be assessed by 

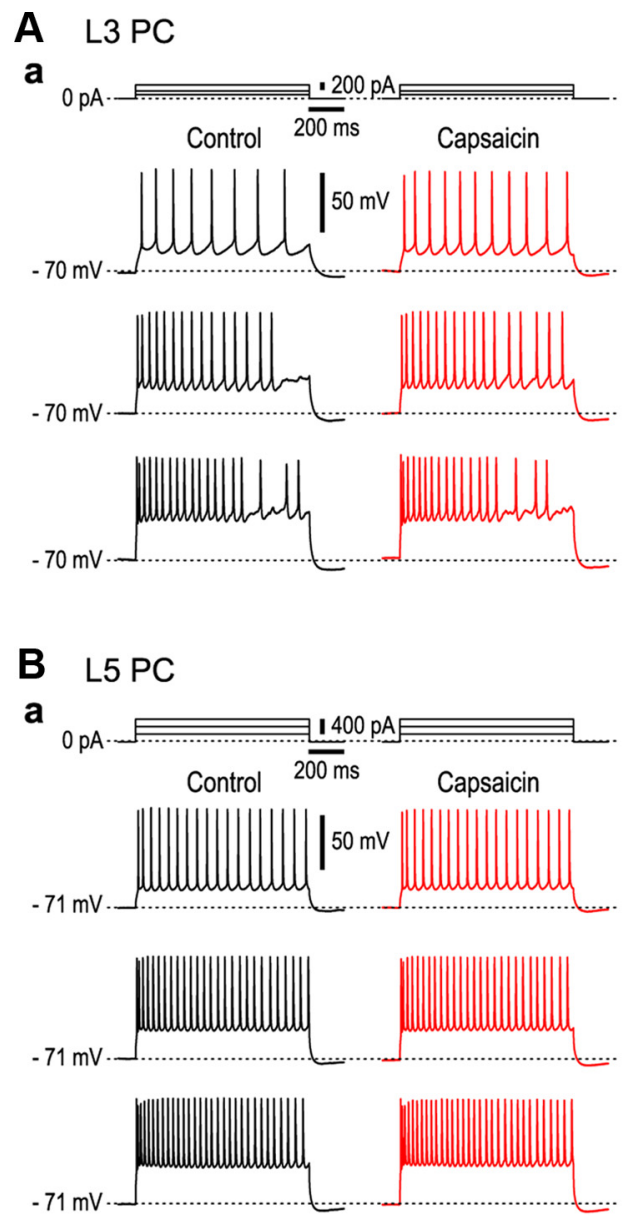
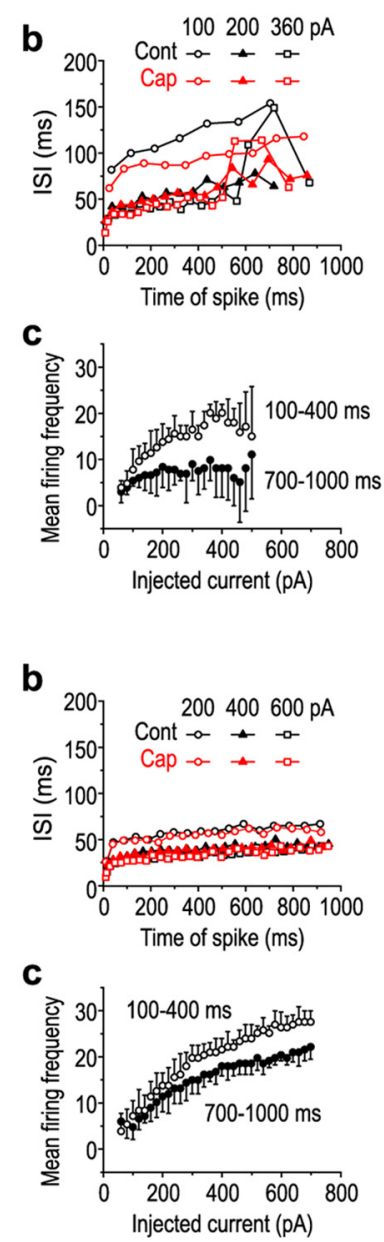
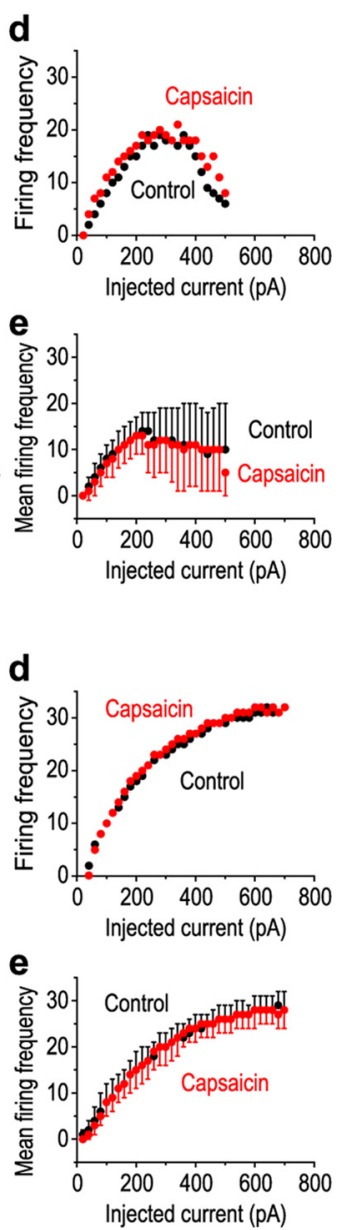

Figure 10. Effects of capsaicin on spike trains induced by current pulse injections. $\mathbf{A a}, \mathbf{B a}$, Sample traces of spike trains induced in an $\mathrm{L} 3 \mathrm{PC}(\boldsymbol{A a})$ and in an $\mathrm{L} 5 \mathrm{PC}(\boldsymbol{B a})$ by injection of current pulses with intensities of 100,200 , and $360 \mathrm{pA}$ and with intensities of 200,400 , and $600 \mathrm{pA}$ (top), respectively, in the absence (black traces) and presence (red traces) of $5 \mu \boldsymbol{M}$ capsaicin. $\boldsymbol{A} \boldsymbol{b}, \boldsymbol{B} \boldsymbol{b}, \mathrm{Plots}$ of ISIs of the spike trains in the L3 and L5 PCS (shown in $A \boldsymbol{a}$ and $B \boldsymbol{a}$, respectively) against the time after the onset of current pulse with respective intensities. $A \boldsymbol{c}, \boldsymbol{B} \boldsymbol{c}$, Relationships between the mean ( \pm SD) firing frequencies and intensities of current pulses obtained during the time periods of $100-400 \mathrm{~ms}$ (open circles) and $700-1000 \mathrm{~ms}$ (filled circles) after the onset of the current pulse in the $L 3$ ( $n=$ $5 ; A c)$ and $L 5 P C s(n=5 ; B \boldsymbol{c})$. Ad, $B \boldsymbol{d}$, Relationships between firing frequencies and intensities of current pulses obtained during the entire duration of current pulses in the $L 3$ and $L 5 P C s$ (shown in $A \boldsymbol{a}$ and $\boldsymbol{B a}$, respectively) in the absence (black circles) and presence (red circles) of capsaicin. $\boldsymbol{A} \boldsymbol{e}, \boldsymbol{B} \boldsymbol{e}$, Relationship between the mean ( $\pm S D)$ firing frequencies and intensities of current pulses obtained during the entire duration of current pulses in $\mathrm{L} 3(n=5 ; A e)$ and $\mathrm{L} 5 \mathrm{PCs}(n=5 ; B e)$ in the absence (black circles) and presence (red circles) of capsaicin.

the difference between the mean spike frequency during the time section of 100-400 $\mathrm{ms}$ and that during 700-1000 ms (Fig. $10 A c, B c)$. The L3 PCs displayed prominent differences at any current intensities (Fig. 10Ac), whereas the L5 PCs displayed little differences up to 220-230 pA and thereafter showed differences (Fig. $10 B c$ ). The differences observed at the current intensities ranging between 300 and $500 \mathrm{pA}$ were significantly larger $\left({ }^{\$} p<\right.$ $0.04)$ in L3 PCs $(n=6)$ than that in L5 PCs $(n=5)$. With an increase in the intensity of injected currents, the mean firing frequency in L3 PCs increased but soon reached a maximum plateau $(18 \pm 4 \mathrm{~Hz})$ at $200-500 \mathrm{pA}$ (Fig. $10 \mathrm{Ae}$ ) and occasionally decreased thereafter (Fig. 10Ad); however, that in L5 PCs increased to $28 \pm 3 \mathrm{~Hz}$ at $600 \mathrm{pA}$ (Fig. $10 \mathrm{Bd}, \mathrm{Be}$ ). These observations suggest that the excitability of L5 PCs is $\sim 1.6$ times higher than that of L3 PCs. Following application of $5 \mu \mathrm{M}$ capsaicin, the resting membrane potential was significantly depolarized from $-70 \pm 3$ to $-67 \pm 3 \mathrm{mV}$ in the L3 PCs $\left({ }^{\ddagger} p<0.001 ; n=6\right)$ but remained almost unchanged in the L 5 PCs $(-69 \pm 1$ vs $-69 \pm 2$ $\left.\mathrm{mV} ;{ }^{\ddagger} p>0.2 ; n=5\right)$. The two distinct firing patterns caused in L3 and L5 PCs by current pulse injections remained virtually unchanged with the application of capsaicin. There was no signifi- cant (L3, $\left.{ }^{\S} p>0.8 ; \mathrm{L} 5,{ }^{\S} p>0.9\right)$ difference in the mean firing frequency before and after the application of capsaicin in L3 and L5 PCs (Fig. $10 \mathrm{Ae}, \mathrm{Be}$ ). However, this result does not preclude the possibility that capsaicin modulates spike firing evoked by synaptic activation.

\section{Capsaicin modulates spike firings evoked by synaptic activation}

In response to $\mathrm{L} 4$ stimulation $(2-3 \mu \mathrm{A})$ that causes only local optical responses instead of a columnar spatial pattern in Gu-I, an L3 PC displayed a transient burst of spikes riding over the slow depolarization envelope (Fig. $11 A a$, top, ${ }^{\star}$ ). In the presence of 5 $\mu \mathrm{M}$ capsaicin, the same stimulation induced a similar transient burst of spikes $\left(^{*}\right)$ and subsequent spike firings that appeared to be triggered from vigorous bombardments of EPSPs (Fig. 11 $A b$, top), as shown in the enlarged trace (Fig. $11 A b$, bottom). Such vigorous bombardments of EPSPs were consistently observed only in the presence of capsaicin in the five L3 PCs examined. The mean number of spikes and the mean spike frequency measured during the capsaicin-induced additional spike firings were $9 \pm 4$ and $4.4 \pm 2.4 \mathrm{~Hz}$, respectively $(n=5)$ (Fig. $11 A c$, gray area). In 
contrast, in the five L5 PCs examined, capsaicin neither induced EPSP bombardment nor depolarized the resting membrane potential, but enhanced the transient burst firing evoked by L4 stimulation (Fig. 11, compare $B a, B b$ ). The mean number of spikes measured during the transient burst firing was significantly $\left({ }^{\ddagger} p<0.05, n=5\right)$ increased from $9 \pm 2$ to $15 \pm 3$, while the mean spike frequency was insignificantly ( ${ }^{*} p>0.1, n=5$ ) increased from $13 \pm 4$ to $18 \pm 3 \mathrm{~Hz}$ (Fig. $11 B c)$. Such an enhancement was pronounced at the first stimulation but progressively declined in response to subsequent stimulations, presumably due to the tachyphylaxis of TRPV1 responses. Thus, transient bursts induced by a weak L4 stimulation (2-3 $\mu \mathrm{A})$ were either prolonged in L3 or enhanced in L5 PCs by capsaicin, but only to $<2-3 \mathrm{~s}$.

\section{Capsaicin induces enormously sustained rhythmic firings at $4 \mathrm{~Hz}$ in L3 PCs}

We next addressed what firing patterns govern L3 and L5 PCs during the oscillatory optical response triggered by stimulation of $\mathrm{L} 4$ in $\mathrm{Gu}-\mathrm{I}$ at $4-6 \mu \mathrm{A}$ in the presence of capsaicin (Figs. 5-7). In response to stimulation of $\mathrm{L} 4$ in $\mathrm{Gu}-\mathrm{I}$ at $4-6 \mu \mathrm{A}$ in the presence of capsaicin, sustained firings with varying durations were induced in a total of $75 \mathrm{~L} 3 \mathrm{PCs}$ located within the stimulated column (Fig. $12 \mathrm{Ba}$ ). The cumulative frequency histogram of durations of sustained firings observed in all the L3 PCs displayed a steep increase followed by a much slower increase (Fig. 12 Aa, black continuous curve). A curve fitting (red dotted curve) of the cumulative frequency histogram with an integration of the sum of two normal distribution functions revealed the presence of short and long duration groups, which were represented as the two distinct cumulative probability distributions (K-S test, $p<0.001$; Fig. $12 \mathrm{Aa}$, black dotted and interrupted curves, respectively). The L3 PCs of the long duration group thus determined $(n=24)$ displayed enormously sustained spike firings ( $\geq 108 \mathrm{~s}$; Fig. $12 \mathrm{Ab}$, gray columns), which persisted for $311 \pm 124 \mathrm{~s}$ (Fig. $12 \mathrm{Ba}, \mathrm{D}$ ). High-amplitude oscillatory fluctuations of membrane potential (Fig. $12 \mathrm{Ba},{ }^{*}$ ) were usually observed after the cessation of the sustained spike firing, suggesting the occurrence of vigorous bombardments of EPSPs, similar to the results shown in Figure $11 A b$. In 10 of the 24 L3 PCs constituting the long-duration group, sustained spike firings were rhythmic as shown on an expanded timescale (Fig. 12 Bb), and persisted for $351 \pm 170 \mathrm{~s}$. The autocorrelogram of the entire spike train (Fig. $12 \mathrm{Ba}$ ) revealed that the spike activity occurred with a periodicity of $0.26 \pm 0.04 \mathrm{~s}(n=10$; Fig. $12 \mathrm{Bc})$. This periodicity was more clearly observed in the early phase of sustained spike firings (Fig. $12 \mathrm{Ba}$, blue bar; $B d$ ).

However, clear periodicity was not observed in most of the 51 L3 PCs constituting the short-duration group $(28 \pm 18 \mathrm{~s}$; Fig. $12 \mathrm{Ab}$, hollow columns; $\mathrm{D})$. This observation suggests that the duration of sustained firings would be more prolonged as periodicity becomes more apparent. Thus, the rhythmic firing may be sustained depending on the strength of synaptic connectivity between the recorded L3 PC and surrounding PCs that dis- play network oscillatory activities. Not only the duration of sustained firings in the long group but also that in the short group L3 PCs was significantly longer $\left({ }^{\dagger} p<0.001\right)$ than that observed in the absence of capsaicin $(9 \pm 4 \mathrm{~s} ; n=11$; Fig. $12 C a, D)$, and also significant longer $\left({ }^{\dagger} p<0.001\right)$ than that observed after coapplication of capsaicin with $0.5 \mu \mathrm{M}$ I-RTX $(11 \pm 6 \mathrm{~s} ; n=14$; Fig. $12 \mathrm{Cb}, \mathrm{D})$. Thus, the sustained rhythmic firing at $4.0 \pm 0.6 \mathrm{~Hz}(n=$ 10) observed in L3 PCs is likely to be induced through the activation of TRPV1, consistent with the F4 component observed in the oscillatory optical responses (Fig. $8 \mathrm{Ba}$, blue curve).

\section{Capsaicin induces short-lived sustained rhythmic firings at 8 $\mathrm{Hz}$ in $\mathrm{L} 5 \mathrm{PCs}$}

Stimulation of L4 in the presence of capsaicin induced sustained firings with varying durations in a total of 21 L5 PCs located within the stimulated column. Similar to the case with L3 PCs (Fig. 12A), a curve fitting of the cumulative frequency histogram of durations of sustained firings revealed the presence of two subgroups; the short-lived ( $49 \pm 20 s ; n=9)$ and transient $(11 \pm$ $3 \mathrm{~s} ; n=12$ ) groups (Fig. 13A,F). In six of the nine L5 PCs constituting the short-lived group, sustained firings were rhythmic with a mean periodicity of $0.13 \pm 0.01 \mathrm{~s}$ (Fig. 13Ba-Bc), which was significantly shorter $\left({ }^{\dagger} p<0.001\right)$ than that observed in L3 PCs (Fig. $12 B c, B d$ ). However, periodicity was not clearly observed in the 12 L5 PCs constituting the transient group. The sustained firing persisted only for $7 \pm 3 \mathrm{~s}(n=5)$ in the absence of capsaicin (Fig. 13C), which was significantly shorter $\left({ }^{\dagger} p<0.001\right.$ and ${ }^{\dagger} p<0.03$ ) than those in the short-lived and transient groups (Fig. 13F). Thus, the short-lived sustained and rhythmic firings at $8.0 \pm 0.7 \mathrm{~Hz}(n=6)$ observed in the L5 PCs is likely to be induced through the activation of TRPV1, consistent with the F8 component (Fig. $8 \mathrm{Ba}$ ). The duration of sustained firings in the shortlived group in the L5 PCs was approximately six times shorter 
$\mathbf{A}_{\mathbf{a}}$

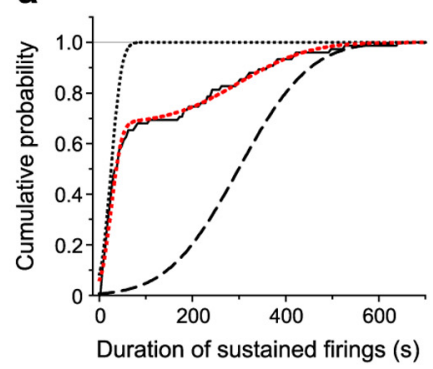

B a

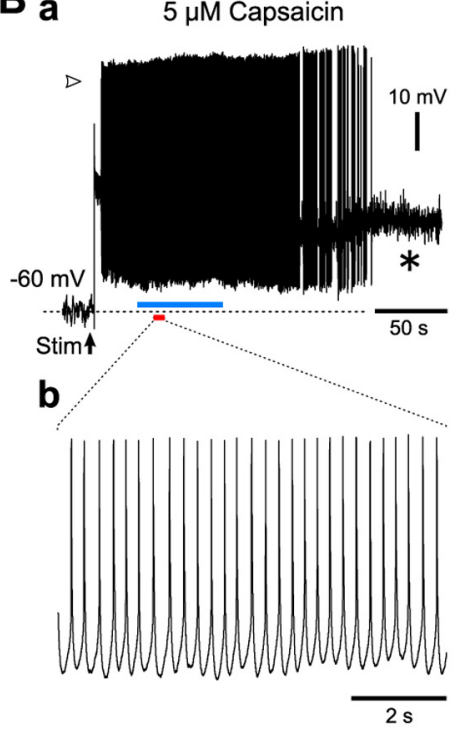

b

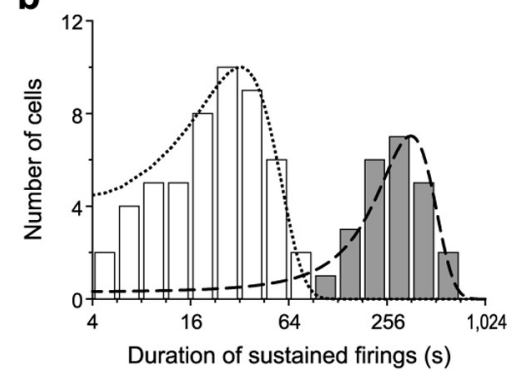

C

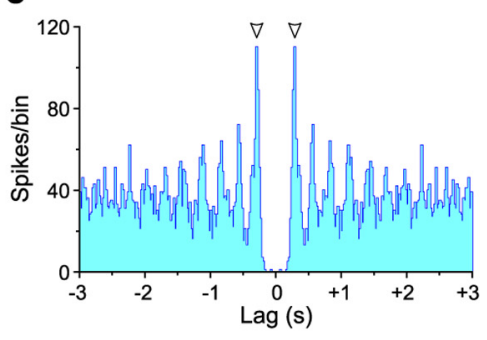

d

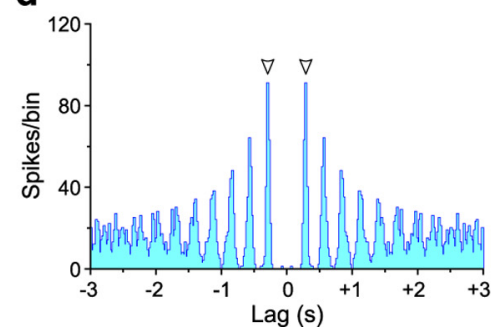

C

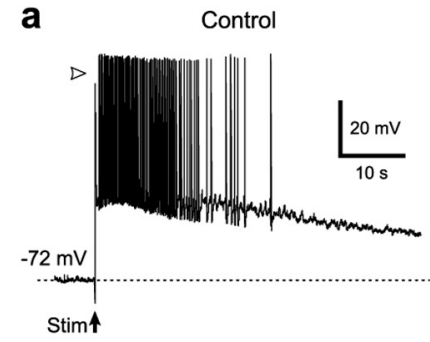

b $\quad 5 \mu M$ Capsaicin + I-RTX
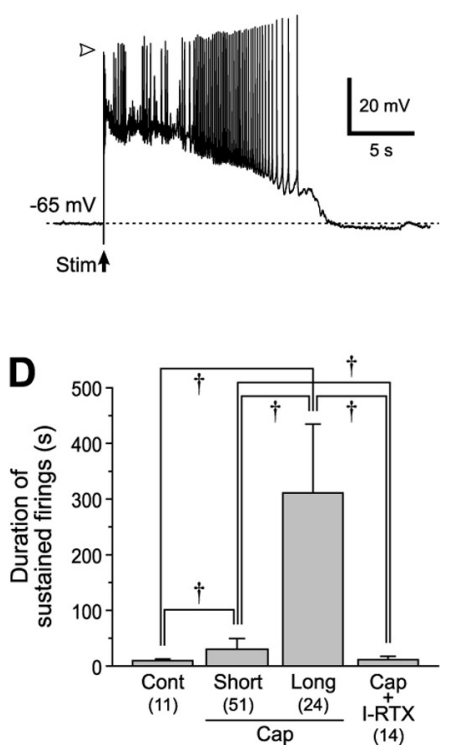

Figure 12. Sustained spike firings in L3 PCS. A, A cumulative frequency histogram of durations of capsaicin-induced sustained firings observed in $75 \mathrm{~L} 3 \mathrm{PCS}(\boldsymbol{a})$. The black continuous and red dotted curves represent a cumulative frequency histogram and its approximation by a cumulative distribution function of the sum of two normal distributions, respectively. The black dotted and interrupted curves represent the cumulative probability distributions in the short and long duration groups, respectively, between which there was a significant difference (K-S test, $p<0.001)$. A histogram of firing durations with a logarithmic bin width; $(\sqrt{2})^{i+4}-(\sqrt{2})^{i+3}$ s for ith bin $(\boldsymbol{b})$. The hollow and gray columns represent the short- and long-duration groups, respectively. The two normal distributions were illustrated with the same curves as those applied in $\boldsymbol{a}$. B. Sustained spike firings induced in an L3 PC evoked by $6 \mu \mathrm{A} L 4$ stimulation in the presence of $5 \mu \mathrm{m}$ capsaicin $(\boldsymbol{a})$. The asterisk $(*)$ denotes high-amplitude membrane potential fluctuations. The arrowhead indicates a membrane potential level of $0 \mathrm{mV}(\boldsymbol{a})$. Expanded traces of spike firings (red bar, $\boldsymbol{a})(\boldsymbol{b})$. Autocorrelograms of the spike train in the entire period of sustained spike firing (c) and in its early episode (blue bar, $\boldsymbol{a}$ ) (d) showing the same periodicity of $0.29 \mathrm{~s}$ (arrowheads). Bin width, $20 \mathrm{~ms}$. C, Spike firings induced in L3 PCs by stimulation of $\mathrm{L} 4$ with an intensity of $6 \mu \mathrm{A}$ in the absence of capsaicin $(\boldsymbol{a})$ and in the presence of capsaicin and $0.5 \mu \mathrm{M}$ I-RTX (b). The arrowheads indicate a membrane potential level of $0 \mathrm{mV}$. D, Mean ( + SD) burst durations obtained in L3 PCs under the respective conditions. ${ }^{\dagger} p<0.001$. Cont, $9 \pm 4 \mathrm{~s}(n=11)$; Cap Short, $28 \pm 18 \mathrm{~s}(n=51)$; Cap Long, $311 \pm 124 \mathrm{~s}(n=24) ;$ Cap $+\mathrm{I}-\mathrm{RTX}, 12 \pm 6 \mathrm{~s}(n=14)$.

$\left({ }^{\dagger} p<0.001\right)$ than that in the long group observed in the L3 PCs (Fig. 12D).

Because PMA removed the desensitization of TRPV1 in L5 PCs and enhanced TRPV1 responses in L3 PCs that project to L5 PCs (Fig. 4), PMA would prolong the duration of sustained rhythmic firing in L5 PCs. Indeed, the rhythmic firing induced in the presence of PMA and capsaicin by L4 stimulation persisted for a much longer time $(293 \pm 109 \mathrm{~s})$ in 7 of the 19 L5 PCs examined (Fig. 13Da,F). Unexpectedly, four of the seven L5 PCs examined displayed rhythmic firings with long periodicities $(0.25 \pm 0.02 \mathrm{~s}$; Fig. $13 D b, D c)$, instead of the shorter one seen in the presence of capsaicin alone. In the remaining three L5 PCs, the ISI during the sustained firing increased gradually (Fig. $13 \mathrm{Ea}$ ), and the periodicity also increased from $0.12 \pm 0.02 \mathrm{~s}$ in the initial period $(<200 \mathrm{~s})$ to $\sim 0.25 \pm 0.02 \mathrm{~s}$ in the late period $(>200 \mathrm{~s})$ (Fig. 13Eb,Ec).

PMA also induced a longer periodicity of $1.35 \pm 0.09 \mathrm{~s}(n=5)$ (Fig. $13 D c, E b$, filled arrowhead), which appeared to interfere with shorter periodicities. As the time lag increased toward the peak of the longer periodicity, the peak density of the shorter periodicity was reduced (Fig. $13 D c, E b$ ), presumably due to the disturbance of the short periodicity through interspersed slow firing. These results indicate that L5 PCs display a periodicity of $8.0 \pm 0.7 \mathrm{~Hz}$ in the presence of capsaicin alone, whereas they display altered or additional periodicity of $4.1 \pm 0.3 \mathrm{~Hz}$ together with a further slower one of $0.7 \pm 0.05 \mathrm{~Hz}$ in the presence of PMA and capsaicin.

\section{Discussion}

There is rapidly accumulating evidence that TRPV1 is widely expressed in various brain regions, suggesting its crucial role in brain function (Kauer and Gibson, 2009), but highly restricted distribution of TRPV1 in the CNS has recently been reported as well using Trpv1 reporter mice (Cavanaugh et al., 2011). This discrepancy could be due to the underestimation of reporter molecules attached to TRPV1, which is expressed at a much lower level in the CNS than in the DRG. Although the insular cortices display a higher level of TRPV1 among brain regions, the role of TRPV1 remained unknown. We demonstrated the critical roles 
A

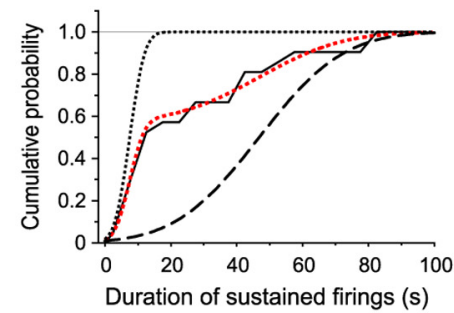

B
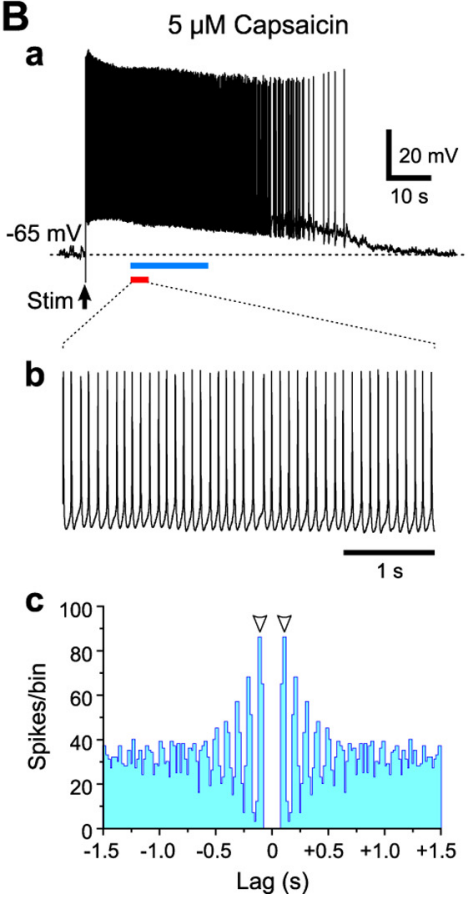

C
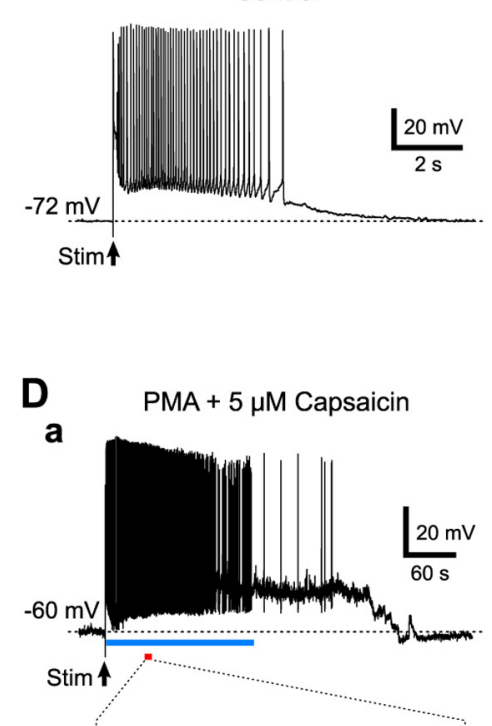

b

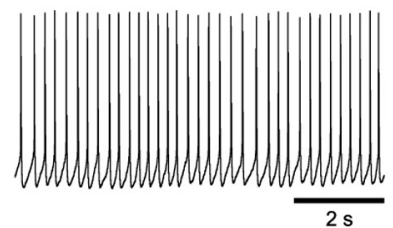

C

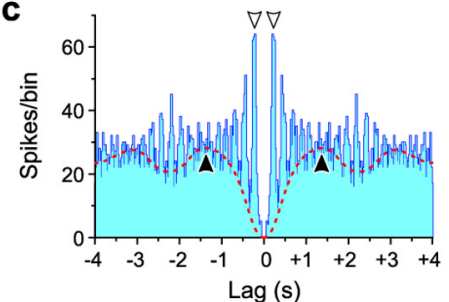

E
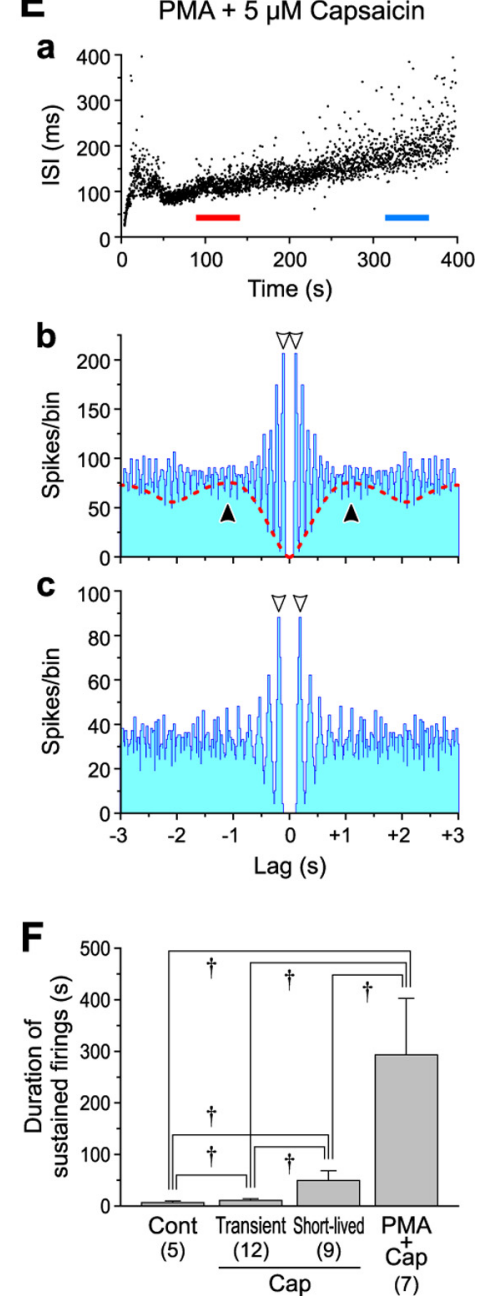

Figure 13. Sustained spike firings in $L 5 P C S$. A, A cumulative frequency histogram of durations of capsaicin-induced sustained firings observed in $21 \mathrm{~L} 5 \mathrm{PCs}$. The black continuous and red dotted curves represent a cumulative frequency histogram and its approximation by a cumulative distribution function of the sum of two normal distributions, respectively. The black dotted and interrupted curves represent the cumulative probability distributions in the short- and long-duration groups, respectively, between which there was a significant difference (K-S test, $p<0.001$ ). $\boldsymbol{B}$, Sustained spike firings induced in an L5PC evoked by $6 \mu \mathrm{A} \mathrm{L} 4$ stimulation in the presence of $5 \mu \mathrm{m}$ capsaicin (a). Expanded traces of spike firings (red bar, $\boldsymbol{a})(\boldsymbol{b})$. Autocorrelogram of the spike train (blue bar, $\boldsymbol{a}$ ) showing a periodicity of $0.11 \mathrm{~s}$ (arrowheads) (c). Bin width, $20 \mathrm{~ms}$. C, Spike firings induced in an L5PC evoked by $6 \mu A$ L 4 stimulation in the absence of capsaicin. $\boldsymbol{D}$, Sustained spike firings induced in an L5 PC evoked by $6 \mu \mathrm{AL}$ stimulation in the presence of PMA and capsaicin $(\boldsymbol{a})$. An expanded trace of spike firings (red bar, $\boldsymbol{a})(\boldsymbol{b})$. Autocorrelogram of the spike train (blue bar, $\boldsymbol{a})$ showing two periodicities of 0.23 (open arrowheads) and $1.3 \mathrm{~s}$ (filled arrowheads) (c). Bin width, $20 \mathrm{~ms}$. $E$, Plot of ISIs during sustained spike firing against the time after stimulation in an L5 PC evoked in the presence of PMA and capsaicin $(\boldsymbol{a})$. Autocorrelograms of the spike trains (red and blue bars, $\boldsymbol{a}$ ) showing shorter $(0.13 \mathrm{~s}, \boldsymbol{b})$ and longer $(0.25 \mathrm{~s}, \boldsymbol{c})$ periodicities (open arrowheads). The autocorrelogram $(\boldsymbol{b})$ also revealed a further longer periodicity of $\sim 1 \mathrm{~s}$ (filled arrowheads) in the L5 PC. Bin width, $20 \mathrm{~ms}$. $\boldsymbol{F}$, Mean (+SD) burst durations obtained in L5 PCs under respective conditions. ${ }^{\dagger} p<0.03$. Cont, $7 \pm 3 s(n=5)$; Cap Transient, $11 \pm 3 s(n=12)$; Cap Short-lived, $49 \pm 20 \mathrm{~s}(n=9)$; PMA + Cap, $293 \pm 109 \mathrm{~s}(n=7)$.

of TRPV1 in inducing theta-band synchronization between the gustatory and autonomic insular cortices.

\section{Possible columnar integration in Gu-I and its modulation by capsaicin}

It has been reported that, during taste administrations in rats, Gu-I displays network oscillations at 7-12 Hz persisting for $\sim 2 \mathrm{~s}$ (Tort et al., 2010) and distinct spatial patterns of neural ensemble activities over a large cortical region $\left(\sim 3 \mathrm{~mm}^{2}\right)$ depending on taste quality (Yamamoto, 1987; Accolla et al., 2007). In the present study, in the horizontal slice preparations of Gu-I, the stimulation of $\mathrm{L} 4$ at $1.2 \times \mathrm{Th}^{\star}$ caused stereotyped optical responses, which displayed monophasic temporal profiles with halfdurations of $91 \pm 21 \mathrm{~ms}$ (Fig. $5 A b$ ) and columnar spatial profiles with half-widths of $588 \pm 49 \mu \mathrm{m}$ (Fig. $5 D b ; n=5$ ). These temporal and spatial profiles were similar $\left({ }^{\dagger} p>0.1\right.$ and $\left.{ }^{\dagger} p>0.3\right)$ to those (half-durations, $71 \pm 20 \mathrm{~ms}$; half-widths, $491 \pm 83 \mu \mathrm{m}$; $n=14)$ obtained by stimulation at $1.2 \times \mathrm{Th}^{\star}$ in the coronal slice preparations of Gu-I [partly from the study by Sato et al. (2008)], in which the presence of the functional column as a unitary local circuit was firmly established as follows: Stimulation of L3 or L5 in the presence of picrotoxin as well as that of L4 in the absence of picrotoxin caused the excitation that invariably spreads vertically across layers uniformly [Sato et al. (2008), their Figs. 8, 9], thus inducing a stereotyped columnar spatial pattern, regardless of the stimulation site in Gu-I. Although the columnar spatial pattern may not necessarily reflect the classically defined functional column such as orientation or ocular dominance columns (Hubel and Wiesel, 1962), both types of columns serve as a functional unit. Such temporal and spatial profiles of excitation spreads, evoked by electrical stimulation, were much shorter and narrower than the temporal and spatial profiles of responses to taste stimulations (Yamamoto, 1987; Accolla et al., 2007), suggesting that taste stimulations cause distinct integrations among several 
A

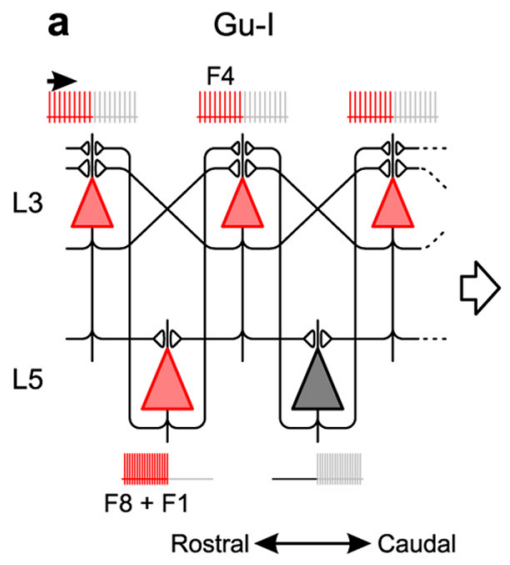

B
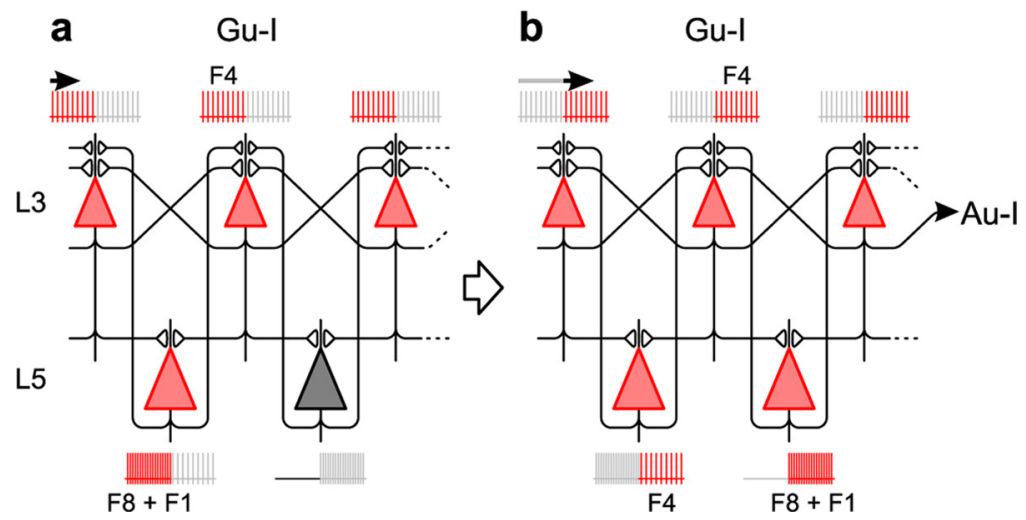

Figure 14. Possible network for theta-band synchronization. $\boldsymbol{A}$, Sustained rhythmic firing at $4 \mathrm{~Hz}$ in $\mathrm{L} 3 \mathrm{PCs}$ generated by recurrent network among $L 3 P$ Cs in the presence of capsaicin. A short-lived rhythmic firing at 8 Hz occurs alternately in adjacent $L 5$ PCs during the sustained rhythmic firing in L3 PCs. Such network oscillations propagate from Gu-I to Au-I. Red and gray represent the present and future $(\boldsymbol{a})$ or the present and past $(\boldsymbol{b})$ activities, respectively. $\boldsymbol{B}$, Enhancement of recurrent network synchronization among $L 3$ PCs by coapplication of PMA and capsaicin engages $L 5 P C$ in the same theta-band synchronization after a short-lived 8 $\mathrm{Hz}$ firing. Red and gray represent the present and future $(\boldsymbol{a})$ or the present and past $(\boldsymbol{b})$ activities, respectively.

are consistent with the natures of type I and type II theta oscillations, respectively, and are consistent with the differential firing characteristics of these neurons such that the excitability of L5 PCs was $\sim 1.6$ times higher than that of L3 PCs (Fig. 10).

TRPV1 was mostly detected in the postsynaptic dendritic spines that received asymmetrical synapses in the insular cortex. As there are much less dendritic spines in GABAergic inhibitory neurons (GABA-INs) compared with pyramidal cells, pyramidal cells would be primarily involved in the generation of capsaicininduced theta oscillation. Furthermore, the capsaicin-induced oscillation was not abolished by picrotoxin (figure not shown). However, it is still possible that GABA-INs are involved in the synchronization of pyramidal cells activities, provided that GABA-INs have reciprocal synaptic connections with pyramidal cells that express $\mathrm{GABA}_{\mathrm{B}}$ receptors, as demonstrated in the thalamus (Kim et al., 1997).

The coapplication of capsaicin and PMA altered L5 PCs to display sustained rhythmic firings composed of 1 and $4 \mathrm{~Hz}$ components, instead of or after the shortlived $8 \mathrm{~Hz}$ oscillation (Fig. $13 \mathrm{D}, E$ ). It is possible that an enhancement of the synchronization among L3 PCs by PMA engaged more L5 PCs in the same $4 \mathrm{~Hz}$ oscillation as that in L3 PCs through the interaction between L3 and L5 PCs because the synchronization mechanism among L3 PCs is much more potent than that among L5 PCs (Fig. 14) [Sato et al. (2008), their Fig. 9]. In agreement with this hypothesis, PMA significantly enhanced F4 and F1 components not only in

columns in Gu-I depending on the taste quality. Surprisingly, capsaicin altered the monophasic optical response, confined in a single column, to the oscillatory response at theta-band rhythm, causing synchronization among multiple columns across Gu-I and Au-I (Figs. 5-7). These spatial profiles exceeded those induced by the four basic tastants (Accolla et al., 2007).

\section{Functional coupling between $\mathrm{Gu}-\mathrm{I}$ and $\mathrm{Au}-\mathrm{I}$ by theta oscillation}

Theta oscillations $(4-12 \mathrm{~Hz})$ occur in the hippocampus during memory-guided movement as well as during learning and memory (Hasselmo, 2005). These theta oscillations are often divided into two types (Kramis et al., 1975; Buzsáki, 2002): Type I occurs at slightly higher frequencies $(6-12 \mathrm{~Hz})$ and is associated with motor activity, whereas type II occurs at the lower frequencies $(4-9 \mathrm{~Hz})$ and is associated with the processing of sensory stimuli before initiating motor activity. In the present study, L5 PCs primarily displayed a short-lived $8 \mathrm{~Hz}$ oscillation (Fig. 13 B,F), while L3 PCs mostly displayed a sustained $4 \mathrm{~Hz}$ oscillation (Fig. 12B) in response to stimulations of Gu-I L4 in the presence of capsaicin. In Gu-I and Au-I, as L5 and L3 PCs are involved in motor control and sensory processing, respectively (Yamamoto et al., 1988; Yasui et al., 1991; Hanamori et al., 1998), $8 \mathrm{~Hz}$ oscillation in L5 PCs and $4 \mathrm{~Hz}$ oscillation in L3 PCs
L3 but also in L5 in Gu-I (Fig. 9Ba, Ca). These findings are consistent with the fact that both L3 and L5 PCs have their presynaptic terminals in both L3 and L5. Together, it is likely that, in L3 or L5, a large fraction of the F4 component and most of the F8 component of oscillatory optical responses reflect synaptic actions evoked in L3 or L5 by the activities of L3 and L5 PCs that fire rhythmically at 4 and $8 \mathrm{~Hz}$, respectively. The F1 component and some remaining fraction of the F4 component are likely to be mediated by the activity of L5 PCs. Au-I stimulation never induced the oscillatory synchronization between Gu-I and Au-I, presumably because the TRPV1 density in L2/3 was five times lower in Au-I than in Gu-I (Fig. 2C).

Oscillations recorded from different brain regions become synchronized to form a large-scale network for the integration of distributed brain function (Varela et al., 2001; Fujisawa and Buzsáki, 2011). Therefore, the theta-band neural synchronization between $\mathrm{Gu}-\mathrm{I}$ and $\mathrm{Au}-\mathrm{I}$ that was demonstrated by cross-power spectral analysis (Fig. 8) might suggest the presence of functional integration between $\mathrm{Gu}-\mathrm{I}$ and $\mathrm{Au}-\mathrm{I}$. Impulse activities arising from TRPV1-expressing terminals in taste buds and oral mucosa (Kido et al., 2003) and those in submucosa in the gastrointestinal tract (Ward et al., 2003) could ascend to layer 4 of Gu-I and Au-I, respectively, through the different thalamic nuclei (Cechetto and 
Saper, 1987). This might result in oscillatory synchronization between Gu-I and Au-I, similar to those observed in the present study. Such synchronization might also be involved in the autonomic responses or, at least, modulate the responses caused by viscero-visceral reflexes. Although the endogenous ligands activating TRPV1 in PCs in the insular cortex are unknown, endocannabinoid may be a candidate for activating TRPV1 (Zygmunt et al., 1999).

\section{Cellular mechanisms for capsaicin response}

In Gu-I, TRPV1 was mostly expressed in the postsynaptic dendritic spines and was two times more densely expressed in L3 than in L5 (Fig. 2C). In L3 PCs, the activation of postsynaptic TRPV1 led to a vigorous bombardment of EPSPs (Fig. 11 Ab), presumably reflecting a reinforcement of the synchronization mechanism among L3 PCs through their reciprocal connections. Subsequently, the prolonged sustained rhythmic firing was induced in L3 PCs by capsaicin (Fig. 12 B). As TRPV1 in L3 PCs did not display $\mathrm{Ca}^{2+}$-dependent desensitization (Fig. $3 A$ ) and was colocalized with asymmetrical synapses (Fig. $2 B$ ), TRPV1-mediated $\mathrm{Ca}^{2+}$ increases might have increased the surface expression of postsynaptic AMPA receptors, similar to the $\mathrm{Ca}^{2+}$-dependent LTP of AMPA responses through NMDA receptor activation (Malenka and Bear, 2004). This idea is consistent with the report that TRPV1 colocalizes with PSD95 (Goswami et al., 2010), similar to the colocalization of NMDA with PSD95 (O’Brien et al., 1998).

In contrast, capsaicin enhanced the transient burst firing evoked in L5 PCs, but seldom induced EPSP bombardment (Fig. $11 \mathrm{Bb}$ ), suggesting that transient $\mathrm{Ca}^{2+}$ influx through TRPV1 enhanced the possible $\mathrm{Ca}^{2+}$-dependent burst (Haj-Dahmane and Andrade, 1997; Kang et al., 1998). Thus, capsaicin differentially modulated spike firings induced in L3 and L5 PCs by L4 stimulation, presumably due to the differential desensitization in capsaicin-induced current between L3 and L5 PCs (Fig. 3). The binding of $\mathrm{Ca}^{2+}$-activated calmodulin to TRPV1 (Numazaki et al., 2003) and the activation of $\mathrm{Ca}^{2+} /$ calmodulin-dependent phosphatase (Jung et al., 2004) mediate the desensitization of TRPV1. In the rat insular cortex, calbindin-28 $\mathrm{kDa}$ was highly expressed in L3 compared with L5 (van Brederode et al., 1991; Paxinos et al., 2008). Therefore, the ability to chelate intracellular free $\mathrm{Ca}^{2+}$ may be much higher in L3 PCs than in L5 PCs, thereby suppressing the $\mathrm{Ca}^{2+}$-dependent desensitization of TRPV1 responses in L3 PCs.

\section{References}

Accolla R, Bathellier B, Petersen CC, Carleton A (2007) Differential spatial representation of taste modalities in the rat gustatory cortex. J Neurosci 27:1396-1404. CrossRef Medline

Buzsáki G (2002) Theta oscillations in the hippocampus. Neuron 33:325340. CrossRef Medline

Caterina MJ, Schumacher MA, Tominaga M, Rosen TA, Levine JD, Julius D (1997) The capsaicin receptor: a heat-activated ion channel in the pain pathway. Nature 389:816-824. CrossRef Medline

Cavanaugh DJ, Chesler AT, Jackson AC, Sigal YM, Yamanaka H, Grant R, O’Donnell D, Nicoll RA, Shah NM, Julius D, Basbaum AI (2011) Trpv1 reporter mice reveal highly restricted brain distribution and functional expression in arteriolar smooth muscle cells. J Neurosci 31:5067-5077. CrossRef Medline

Cechetto DF, Saper CB (1987) Evidence for a viscerotopic sensory representation in the cortex and thalamus in the rat. J Comp Neurol 262:27-45. CrossRef Medline

Cechetto DF, Saper CB (1990) Role of the cerebral cortex in autonomic functions. In: Central regulation of autonomic functions (Loewy AD, Spyer KM, eds), pp 208-223. New York: Oxford UP.

Dunér-Engström M, Fredholm BB, Larsson O, Lundberg JM, Saria A (1986)
Autonomic mechanisms underlying capsaicin induced oral sensations and salivation in man. J Physiol 373:87-96. Medline

Fujisawa S, Buzsáki G (2011) A $4 \mathrm{~Hz}$ oscillation adaptively synchronizes prefrontal, VTA, and hippocampal activities. Neuron 72:153-165. CrossRef Medline

Ganong W (2003) Review of medical physiology, Ed 21. New York: McGraw-Hill Professional.

Goswami C, Rademacher N, Smalla KH, Kalscheuer V, Ropers HH, Gundelfinger ED, Hucho T (2010) TRPV1 acts as a synaptic protein and regulates vesicle recycling. J Cell Sci 123:2045-2057. CrossRef Medline

Grueter BA, Brasnjo G, Malenka RC (2010) Postsynaptic TRPV1 triggers cell type-specific long-term depression in the nucleus accumbens. Nat Neurosci 13:1519-1525. CrossRef Medline

Hachiya S, Kawabata F, Ohnuki K, Inoue N, Yoneda H, Yazawa S, Fushiki T (2007) Effects of CH-19 Sweet, a non-pungent cultivar of red pepper, on sympathetic nervous activity, body temperature, heart rate, and blood pressure in humans. Biosci Biotechnol Biochem 71:671-676. CrossRef Medline

Haj-Dahmane S, Andrade R (1997) Calcium-activated cation nonselective current contributes to the fast afterdepolarization in rat prefrontal cortex neurons. J Neurophysiol 78:1983-1989. Medline

Hanamori T, Kunitake T, Kato K, Kannan H (1998) Responses of neurons in the insular cortex to gustatory, visceral, and nociceptive stimuli in rats. J Neurophysiol 79:2535-2545. Medline

Hasselmo ME (2005) What is the function of hippocampal theta rhythm?Linking behavioral data to phasic properties of field potential and unit recording data. Hippocampus 15:936-949. CrossRef Medline

Hubel DH, Wiesel TN (1962) Receptive fields, binocular interaction and functional architecture in the cat's visual cortex. J Physiol 160:106-154. Medline

Ishida Y, Ugawa S, Ueda T, Murakami S, Shimada S (2002) Vanilloid receptor subtype-1 (VR1) is specifically localized to taste papillae. Brain Res Mol Brain Res 107:17-22. CrossRef Medline

Jasmin L, Burkey AR, Granato A, Ohara PT (2004) Rostral agranular insular cortex and pain areas of the central nervous system: a tract-tracing study in the rat. J Comp Neurol 468:425-440. CrossRef Medline

Jung J, Shin JS, Lee SY, Hwang SW, Koo J, Cho H, Oh U (2004) Phosphorylation of vanilloid receptor 1 by $\mathrm{Ca}^{2+} /$ calmodulin-dependent kinase II regulates its vanilloid binding. J Biol Chem 279:7048-7054. CrossRef Medline

Kang Y, Okada T, Ohmori H (1998) A phenytoin-sensitive cationic current participates in generating the afterdepolarization and burst afterdischarge in rat neocortical pyramidal cells. Eur J Neurosci 10:1363-1375. CrossRef Medline

Kang Y, Dempo Y, Ohashi A, Saito M, Toyoda H, Sato H, Koshino H, Maeda Y, Hirai $\mathrm{T}$ (2007) Nitric oxide activates leak $\mathrm{K}^{+}$currents in the presumed cholinergic neuron of basal forebrain. J Neurophysiol 98:3397-3410. CrossRef Medline

Kauer JA, Gibson HE (2009) Hot flash: TRPV channels in the brain. Trends Neurosci 32:215-224. CrossRef Medline

Kido MA, Muroya H, Yamaza T, Terada Y, Tanaka T (2003) Vanilloid receptor expression in the rat tongue and palate. J Dent Res 82:393-397. CrossRef Medline

Kim S, Kang C, Shin CY, Hwang SW, Yang YD, Shim WS, Park MY, Kim E, Kim M, Kim BM, Cho H, Shin Y, Oh U (2006) TRPV1 recapitulates native capsaicin receptor in sensory neurons in association with Fasassociated factor 1. J Neurosci 26:2403-2412. CrossRef Medline

Kim U, Sanchez-Vives MV, McCormick DA (1997) Functional dynamics of GABAergic inhibition in the thalamus. Science 278:130-134. CrossRef Medline

Koplas PA, Rosenberg RL, Oxford GS (1997) The role of calcium in the desensitization of capsaicin responses in rat dorsal root ganglion neurons. J Neurosci 17:3525-3537. Medline

Kramis R, Vanderwolf CH, Bland BH (1975) Two types of hippocampal rhythmical slow activity in both the rabbit and the rat: relations to behavior and effects of atropine, diethyl ether, urethane, and pentobarbital. Exp Neurol 49:58-85. CrossRef Medline

Lee TS (1954) Physiological gustatory sweating in a warm climate. J Physiol 124:528-542. Medline

Malenka RC, Bear MF (2004) LTP and LTD: an embarrassment of riches. Neuron 44:5-21. CrossRef Medline

Mandadi S, Numazaki M, Tominaga M, Bhat MB, Armati PJ, Roufogalis BD 
(2004) Activation of protein kinase C reverses capsaicin-induced calciumdependent desensitization of TRPV1 ion channels. Cell Calcium 35:471-478. CrossRef Medline

Mandadi S, Tominaga T, Numazaki M, Murayama N, Saito N, Armati PJ, Roufogalis BD, Tominaga M (2006) Increased sensitivity of desensitized TRPV1 by PMA occurs through PKC $\varepsilon$-mediated phosphorylation at S800. Pain 123:106-116. CrossRef Medline

Marinelli S, Di Marzo V, Berretta N, Matias I, Maccarrone M, Bernardi G, Mercuri NB (2003) Presynaptic facilitation of glutamatergic synapses to dopaminergic neurons of the rat substantia nigra by endogenous stimulation of vanilloid receptors. J Neurosci 23:3136-3144. Medline

Numazaki M, Tominaga T, Toyooka H, Tominaga M (2002) Direct phosphorylation of capsaicin receptor VR1 by protein kinase $\mathrm{C} \varepsilon$ and identification of two target serine residues. J Biol Chem 277:13375-13378. CrossRef Medline

Numazaki M, Tominaga T, Takeuchi K, Murayama N, Toyooka H, Tominaga M (2003) Structural determinant of TRPV1 desensitization interacts with calmodulin. Proc Natl Acad Sci U S A 100:8002-8006. CrossRef Medline

O’Brien RJ, Lau LF, Huganir RL (1998) Molecular mechanisms of glutamate receptor clustering at excitatory synapses. Curr Opin Neurobiol 8:364-369. CrossRef Medline

Paxinos G, Watson C (1998) The rat brain in stereotaxic coordinates, Ed 4. Amsterdam: Academic.

Paxinos G, Watson C, Carrive P, Kirkcaldie M, Ashwell K (2008) Chemoarchitectonic atlas of the rat brain, Ed 2. Amsterdam: Academic.

Roberts JC, Davis JB, Benham CD (2004) $\left[{ }^{3} \mathrm{H}\right]$ Resiniferatoxin autoradiography in the CNS of wild-type and TRPV1 null mice defines TRPV1 (VR-1) protein distribution. Brain Res 995:176-183. CrossRef Medline

Rudenga K, Green B, Nachtigal D, Small DM (2010) Evidence for an integrated oral sensory module in the human anterior ventral insula. Chem Senses 35:693-703. CrossRef Medline

Ruggiero DA, Mraovitch S, Granata AR, Anwar M, Reis DJ (1987) A role of insular cortex in cardiovascular function. J Comp Neurol 257:189-207. CrossRef Medline

Sanchez JF, Krause JE, Cortright DN (2001) The distribution and regulation of vanilloid receptor VR1 and VR1 5' splice variant RNA expression in rat. Neuroscience 107:373-381. CrossRef Medline

Sato H, Shimanuki Y, Saito M, Toyoda H, Nokubi T, Maeda Y, Yamamoto T, Kang Y (2008) Differential columnar processing in local circuits of barrel and insular cortices. J Neurosci 28:3076-3089. CrossRef Medline
Sklar B, Hanley J, Simmons WW (1972) An EEG experiment aimed toward identifying dyslexic children. Nature 240:414-416. CrossRef Medline

Tominaga M, Caterina MJ, Malmberg AB, Rosen TA, Gilbert H, Skinner K, Raumann BE, Basbaum AI, Julius D (1998) The cloned capsaicin receptor integrates multiple pain-producing stimuli. Neuron 21:531-543. CrossRef Medline

Tort AB, Fontanini A, Kramer MA, Jones-Lush LM, Kopell NJ, Katz DB (2010) Cortical networks produce three distinct $7-12 \mathrm{~Hz}$ rhythms during single sensory responses in the awake rat. J Neurosci 30:4315-4324. CrossRef Medline

Treede RD, Kenshalo DR, Gracely RH, Jones AK (1999) The cortical representation of pain. Pain 79:105-111. CrossRef Medline

van Brederode JF, Helliesen MK, Hendrickson AE (1991) Distribution of the calcium-binding proteins parvalbumin and calbindin-D28k in the sensorimotor cortex of the rat. Neuroscience 44:157-171. CrossRef Medline

Varela F, Lachaux JP, Rodriguez E, Martinerie J (2001) The brainweb: phase synchronization and large-scale integration. Nat Rev Neurosci 2:229-239. CrossRef Medline

Vellani V, Mapplebeck S, Moriondo A, Davis JB, McNaughton PA (2001) Protein kinase $\mathrm{C}$ activation potentiates gating of the vanilloid receptor VR1 by capsaicin, protons, heat and anandamide. J Physiol 534:813-825. CrossRef Medline

Ward SM, Bayguinov J, Won KJ, Grundy D, Berthoud HR (2003) Distribution of the vanilloid receptor (VR1) in the gastrointestinal tract. J Comp Neurol 465:121-135. CrossRef Medline

Yamamoto T (1987) Cortical organization in gustatory perception. Ann N Y Acad Sci 510:49-54. CrossRef Medline

Yamamoto T, Matsuo R, Kiyomitsu Y, Kitamura R (1988) Sensory inputs from the oral region to the cerebral cortex in behaving rats: an analysis of unit responses in cortical somatosensory and taste areas during ingestive behavior. J Neurophysiol 60:1303-1321. Medline

Yasui Y, Breder CD, Saper CB, Cechetto DF (1991) Autonomic responses and efferent pathways from the insular cortex in the rat. J Comp Neurol 303:355-374. CrossRef Medline

Zygmunt PM, Petersson J, Andersson DA, Chuang H, Sørgård M, Di Marzo V, Julius D, Högestätt ED (1999) Vanilloid receptors on sensory nerves mediate the vasodilator action of anandamide. Nature 400:452-457. CrossRef Medline 